\title{
Smoluchowski-Kramers approximation in the case of variable friction
}

\author{
Mark Freidlin, Wenqing $\mathrm{Hu}^{\dagger}$
}

\begin{abstract}
We consider the small mass asymptotics (Smoluchowski-Kramers approximation) for the Langevin equation with a variable friction coefficient. The limit of the solution in the classical sense does not exist in this case. We study a modification of the Smoluchowski-Kramers approximation. Some applications of the Smoluchowski-Kramers approximation to problems with fast oscillating or discontinuous coefficients are considered.
\end{abstract}

Keywords: Smoluchowski-Kramers approximation, diffusion processes, weak convergence, homogenization.

2010 Mathematics Subject Classification Numbers: 60J60, 60F05, 60H10, 35B27.

\section{Introduction}

The Langevin equation

$$
\mu \ddot{\boldsymbol{q}}_{t}^{\mu}=\boldsymbol{b}\left(\boldsymbol{q}_{t}^{\mu}\right)-\lambda \dot{\boldsymbol{q}}_{t}^{\mu}+\sigma\left(\boldsymbol{q}_{t}^{\mu}\right) \dot{\boldsymbol{W}}_{t}, \boldsymbol{q}_{0}^{\mu}=\boldsymbol{q} \in \mathbb{R}^{n}, \dot{\boldsymbol{q}}_{0}^{\mu}=\boldsymbol{p} \in \mathbb{R}^{n},
$$

describes the motion of a particle of mass $\mu$ in a force field $\boldsymbol{b}(\boldsymbol{q}), \boldsymbol{q} \in \mathbb{R}^{n}$, subjected to random fluctuations and to a friction proportional to the velocity. Here $\boldsymbol{W}_{t}$ is the standard Wiener process in $\mathbb{R}^{n}, \lambda>0$ is the friction coefficient. The vector field $\boldsymbol{b}(\boldsymbol{q})$ and the matrix function $\sigma(\boldsymbol{q})$ are assumed to be continuously differentiable and bounded together with their first derivatives. The matrix $a(\boldsymbol{q})=\left(a_{i j}(\boldsymbol{q})\right)=\sigma(\boldsymbol{q}) \sigma^{*}(\boldsymbol{q})$ is assumed to be non-degenerate.

Put $\boldsymbol{p}_{t}^{\mu}=\dot{\boldsymbol{q}}_{t}^{\mu}$. Then (1.1) can be written as a first order system:

$$
\left\{\begin{array}{l}
\dot{\boldsymbol{q}}_{t}^{\mu}=\boldsymbol{p}_{t}^{\mu} \\
\dot{\boldsymbol{p}}_{t}^{\mu}=\frac{1}{\mu} \boldsymbol{b}\left(\boldsymbol{q}_{t}^{\mu}\right)-\frac{\lambda}{\mu} \boldsymbol{p}_{t}^{\mu}+\frac{1}{\mu} \sigma\left(\boldsymbol{q}_{t}^{\mu}\right) \dot{\boldsymbol{W}}_{t}
\end{array}\right.
$$

\footnotetext{
${ }^{*}$ Dept of Mathematics, University of Maryland at College Park, mif@math.umd.edu.

${ }^{\dagger}$ Dept of Mathematics, University of Maryland at College Park, huwenqing@math.umd.edu.
} 
The diffusion process $\left(\boldsymbol{p}_{t}^{\mu}, \boldsymbol{q}_{t}^{\mu}\right)=\boldsymbol{X}_{t}^{\mu}$ in $\mathbb{R}^{2 n}$ is governed by the generator $L$ :

$$
L u(\boldsymbol{p}, \boldsymbol{q})=\frac{1}{2 \mu^{2}} \sum_{i, j=1}^{n} a_{i j}(\boldsymbol{q}) \frac{\partial^{2} u}{\partial p_{i} \partial p_{j}}+\frac{1}{\mu}(\boldsymbol{b}(\boldsymbol{q})-\lambda \boldsymbol{p}) \cdot \nabla_{\boldsymbol{p}} u+\boldsymbol{p} \cdot \nabla_{\boldsymbol{q}} u .
$$

Note that, since functions $\boldsymbol{q}_{t}^{\mu}$ are continuously differentiable with probability one,

$$
\int_{0}^{t} \sigma_{i j}\left(\boldsymbol{q}_{s}^{\mu}\right) d W_{s}^{j}=\sigma_{i j}\left(\boldsymbol{q}_{t}^{\mu}\right) W_{t}^{j}-\int_{0}^{t} W_{s}^{j}\left(\nabla_{\boldsymbol{q}} \sigma_{i j}\left(\boldsymbol{q}_{s}^{\mu}\right) \cdot \boldsymbol{p}_{s}^{\mu}\right) d s .
$$

This allows to consider equations (1.2) for each trajectory $\boldsymbol{W}_{t}$ individually, and there is no necessity in the introduction of a stochastic integral. In particular, if (1.2) is considered as a stochastic differential equation, stochastic integrals in the Itô and in the Stratonovich sense coincide: $\int_{0}^{t} \sigma\left(\boldsymbol{q}_{s}^{\mu}\right) d \boldsymbol{W}_{s}=\int_{0}^{t} \sigma\left(\boldsymbol{q}_{s}^{\mu}\right) \circ d \boldsymbol{W}_{s}$.

It is assumed usually that the friction coefficient $\lambda$ is constant. Under this assumption, one can prove that $\boldsymbol{q}_{t}^{\mu}$ converges in probability as $\mu \downarrow 0$ uniformly on each finite time interval $[0, T]$ to an $n$-dimensional diffusion process $\boldsymbol{q}_{t}$ : for any $\kappa, T>0$ and any $\boldsymbol{p}_{0}^{\mu}=\boldsymbol{p} \in \mathbb{R}^{n}$ fixed,

$$
\lim _{\mu \downarrow 0} \mathbf{P}\left(\max _{0 \leq t \leq T}\left|\boldsymbol{q}_{t}^{\mu}-\boldsymbol{q}_{t}\right|_{\mathbb{R}^{d}}>\kappa\right)=0 .
$$

Here $\boldsymbol{q}_{t}$ is the solution of equation

$$
\dot{\boldsymbol{q}}_{t}=\frac{1}{\lambda} \boldsymbol{b}\left(\boldsymbol{q}_{t}\right)+\frac{1}{\lambda} \sigma\left(\boldsymbol{q}_{t}\right) \dot{\boldsymbol{W}}_{t}, \boldsymbol{q}_{0}=\boldsymbol{q}_{0}^{\mu}=\boldsymbol{q} \in \mathbb{R}^{n} .
$$

The stochastic term in (1.3) should be understood in the Itô sense.

The approximation of $\boldsymbol{q}_{t}^{\mu}$ by $\boldsymbol{q}_{t}$ for $0<\mu<<1$ is called the Smoluchowski-Kramers approximation. This is the main justification for replacement of the second order equation (1.1) by the first order equation (1.3). The price for such a simplification, in particular, consists of certain non-universality of equation (1.3): The white noise in (1.1) is an idealization of a more regular stochastic process $\dot{\boldsymbol{W}}_{t}^{\delta}$ with correlation radius $\delta<<1$ converging to $\dot{\boldsymbol{W}}_{t}$ as $\delta \downarrow 0$. Let $\boldsymbol{q}_{t}^{\mu, \delta}$ be the solution of equation (1.1) with $\dot{\boldsymbol{W}}_{t}$ replaced by $\dot{\boldsymbol{W}}_{t}^{\delta}$. Then limit of $\boldsymbol{q}_{t}^{\mu, \delta}$ as $\mu, \delta \downarrow 0$ depends on the relation between $\mu$ and $\delta$. Say, if first $\delta \downarrow 0$ and then $\mu \downarrow 0$, the stochastic integral in (1.3) should be understood in the Itô sense; if first $\mu \downarrow 0$ and then $\delta \downarrow 0, \boldsymbol{q}_{t}^{\mu, \delta}$ converges to the solution of (1.3) with stochastic integral in the Stratonovich sense. (See, for instance, [5].)

Consider now the case of a variable friction coefficient $\lambda=\lambda(\boldsymbol{q})$. We assume that $\lambda(\boldsymbol{q})$ has continuous bounded derivatives and $0<\lambda_{0} \leq \lambda(\boldsymbol{q}) \leq \Lambda<\infty$. It turns out, as we will see in the next section, that in this case the solution $\boldsymbol{q}_{t}^{\mu}$ of (1.1) does not converge, in general, to the solution of (1.3) with $\lambda=\lambda(\boldsymbol{q})$, so that the SmoluchowskiKramers approximation should be modified. In order to do this, we consider equation (1.1) with $\dot{\boldsymbol{W}}_{t}$ replaced by $\dot{\boldsymbol{W}}_{t}^{\delta}$ described above: 


$$
\mu \ddot{\boldsymbol{q}}_{t}^{\mu, \delta}=\boldsymbol{b}\left(\boldsymbol{q}_{t}^{\mu, \delta}\right)-\lambda\left(\boldsymbol{q}_{t}^{\mu, \delta}\right) \dot{\boldsymbol{q}}_{t}^{\mu, \delta}+\sigma\left(\boldsymbol{q}_{t}^{\mu, \delta}\right) \dot{\boldsymbol{W}}_{t}^{\delta}, \boldsymbol{q}_{0}^{\mu, \delta}=\boldsymbol{q}, \dot{\boldsymbol{q}}_{0}^{\mu, \delta}=\boldsymbol{p} .
$$

We prove that after such a regularization, the solution of (1.4) has a limit $\widetilde{\boldsymbol{q}}_{t}^{\delta}$ as $\mu \downarrow 0$, and $\widetilde{\boldsymbol{q}}_{t}^{\delta}$ is the unique solution of the equation obtained from (1.4) as $\mu=0$ :

$$
\dot{\boldsymbol{q}}_{t}^{\delta}=\frac{1}{\lambda\left(\widetilde{\boldsymbol{q}}_{t}^{\delta}\right)} \boldsymbol{b}\left(\widetilde{\boldsymbol{q}}_{t}^{\delta}\right)+\frac{1}{\lambda\left(\widetilde{\boldsymbol{q}}_{t}^{\delta}\right)} \sigma\left(\widetilde{\boldsymbol{q}}_{t}^{\delta}\right) \dot{\boldsymbol{W}}_{t}^{\delta}, \widetilde{\boldsymbol{q}}_{0}^{\delta}=\boldsymbol{q} .
$$

Now we can take $\delta \downarrow 0$ in (1.5). As the result we get the equation

$$
\dot{\boldsymbol{q}}_{t}=\frac{1}{\lambda\left(\widehat{\boldsymbol{q}}_{t}\right)} \boldsymbol{b}\left(\widehat{\boldsymbol{q}}_{t}\right)+\frac{1}{\lambda\left(\widehat{\boldsymbol{q}}_{t}\right)} \sigma\left(\widehat{\boldsymbol{q}}_{t}\right) \circ \dot{\boldsymbol{W}}_{t}, \widehat{\boldsymbol{q}}_{0}=\boldsymbol{q},
$$

where the stochastic term should be understood in the Stratonovich sense. So the regularization leads to a modified Smoluchowski-Kramers equation (1.6). We prove this in Section 3.

Some applications of the Smoluchowski-Kramers approximation are considered in Sections 4 and 5: the case of fast oscillating in the space variable, periodic or stochastic, friction coefficient is studied; gluing condition at the discontinuity points of the friction coefficient are considered. In the last Section 6 , we briefly consider some remarks and generalizations.

Notations. We use $|\bullet|_{\mathbb{R}^{d}}$ to denote the standard Euclidean norm in $\mathbb{R}^{d}$. When $d=1$ we set $|\bullet|_{\mathbb{R}^{1}}=|\bullet|$. For a vector-valued function $\boldsymbol{f}(\boldsymbol{x})=\left(f_{1}(\boldsymbol{x}), \ldots, f_{d}(\boldsymbol{x})\right), \boldsymbol{x} \in \mathbb{R}^{d}$, we set $\|\boldsymbol{f}\|_{\infty}=\max _{1 \leq i \leq d}\left\|f_{i}\right\|_{\infty}=\max _{1 \leq i \leq d} \sup _{\boldsymbol{x} \in \mathbb{R}^{d}}\left|f_{i}(\boldsymbol{x})\right|$. All the vectors are marked with either bold letters or with an arrow on it.

\section{Some estimates. The classical Smoluchowski-Kramers approximation does not work for variable friction coef- ficients}

We consider the following system

$$
\mu \ddot{\boldsymbol{q}}_{t}^{\mu}=\boldsymbol{b}\left(\boldsymbol{q}_{t}^{\mu}\right)-\lambda\left(\boldsymbol{q}_{t}^{\mu}\right) \dot{\boldsymbol{q}}_{t}^{\mu}+\dot{\boldsymbol{W}}_{t}, \boldsymbol{q}_{0}^{\mu}=\boldsymbol{q} \in \mathbb{R}^{d}, \dot{\boldsymbol{q}}_{0}^{\mu}=\boldsymbol{p} \in \mathbb{R}^{d} .
$$

Here $\infty>\Lambda \geq \lambda(\bullet) \geq \lambda_{0}>0$ is a function of $\boldsymbol{q}_{t}^{\mu}$. We assume that function $\lambda(\bullet)$ and the vector field $\boldsymbol{b}(\bullet)$ are continuously differentiable and bounded together with their first derivatives. The process $\boldsymbol{W}_{t}$ is the standard Wiener process in $\mathbb{R}^{d}$. For simplicity of calculations we consider here the case when the diffusion matrix $a(\bullet)$ is the identity 
(compare with (1.1)). The case of general diffusion matrix can be considered in a similar way and we will briefly mention it in Section 6 .

Let $\boldsymbol{p}_{t}^{\mu}=\dot{\boldsymbol{q}}_{t}^{\mu}$, we have, that (2.1) is equivalent to the system

$$
\left\{\begin{array}{l}
\dot{\boldsymbol{q}}_{t}^{\mu}=\boldsymbol{p}_{t}^{\mu} \\
\dot{\boldsymbol{p}}_{t}^{\mu}=\frac{1}{\mu} \boldsymbol{b}\left(\boldsymbol{q}_{t}^{\mu}\right)-\frac{\lambda\left(\boldsymbol{q}_{t}^{\mu}\right)}{\mu} \boldsymbol{p}_{t}^{\mu}+\frac{1}{\mu} \dot{\boldsymbol{W}}_{t}
\end{array}\right.
$$

Then

$\frac{d}{d t}\left(e^{\frac{1}{\mu} \int_{0}^{t} \lambda\left(\boldsymbol{q}_{s}^{\mu}\right) d s} \boldsymbol{p}_{t}^{\mu}\right)=e^{\frac{1}{\mu} \int_{0}^{t} \lambda\left(\boldsymbol{q}_{s}^{\mu}\right) d s}\left(\dot{\boldsymbol{p}}_{t}^{\mu}+\frac{1}{\mu} \lambda\left(\boldsymbol{q}_{t}^{\mu}\right) \boldsymbol{p}_{t}^{\mu}\right)=e^{\frac{1}{\mu} \int_{0}^{t} \lambda\left(\boldsymbol{q}_{s}^{\mu}\right) d s}\left(\frac{1}{\mu} \boldsymbol{b}\left(\boldsymbol{q}_{t}^{\mu}\right)+\frac{1}{\mu} \dot{\boldsymbol{W}}_{t}\right)$,

and

$$
e^{\frac{1}{\mu} \int_{0}^{t} \lambda\left(\boldsymbol{q}_{s}^{\mu}\right) d s} \boldsymbol{p}_{t}^{\mu}-\boldsymbol{p}=\frac{1}{\mu} \int_{0}^{t} e^{\frac{1}{\mu} \int_{0}^{s} \lambda\left(\boldsymbol{q}_{r}^{\mu}\right) d r} \boldsymbol{b}\left(\boldsymbol{q}_{s}^{\mu}\right) d s+\frac{1}{\mu} \int_{0}^{t} e^{\frac{1}{\mu} \int_{0}^{s} \lambda\left(\boldsymbol{q}_{r}^{\mu}\right) d r} d \boldsymbol{W}_{s} .
$$

For notational convenience we introduce the function $A(\mu, t)=\int_{0}^{t} \lambda\left(\boldsymbol{q}_{s}^{\mu}\right) d s$. It is clear that $t \Lambda \geq A(\mu, t) \geq t \lambda_{0}$. Using (2.3) we have, that

$$
\boldsymbol{p}_{t}^{\mu}=e^{-\frac{1}{\mu} A(\mu, t)}\left(\boldsymbol{p}+\frac{1}{\mu} \int_{0}^{t} e^{\frac{1}{\mu} A(\mu, s)} \boldsymbol{b}\left(\boldsymbol{q}_{s}^{\mu}\right) d s+\frac{1}{\mu} \int_{0}^{t} e^{\frac{1}{\mu} A(\mu, s)} d \boldsymbol{W}_{s}\right) .
$$

Therefore we have

$$
\begin{aligned}
& \boldsymbol{q}_{t}^{\mu}=\boldsymbol{q}+\int_{0}^{t} \boldsymbol{p}_{s}^{\mu} d s \\
& =\boldsymbol{q}+\boldsymbol{p} \int_{0}^{t} e^{-\frac{1}{\mu} A(\mu, s)} d s+\frac{1}{\mu} \int_{0}^{t} e^{-\frac{1}{\mu} A(\mu, s)}\left(\int_{0}^{s} e^{\frac{1}{\mu} A(\mu, r)} \boldsymbol{b}\left(\boldsymbol{q}_{r}^{\mu}\right) d r\right) d s+ \\
& +\frac{1}{\mu} \int_{0}^{t} e^{-\frac{1}{\mu} A(\mu, s)}\left(\int_{0}^{s} e^{\frac{1}{\mu} A(\mu, r)} d \boldsymbol{W}_{r}\right) d s \\
& =\boldsymbol{q}+\boldsymbol{\alpha}(\mu)+\boldsymbol{\beta}(\mu)+\gamma(\mu) \text {. }
\end{aligned}
$$

Here $\boldsymbol{\alpha}(\mu), \boldsymbol{\beta}(\mu), \boldsymbol{\gamma}(\mu)$ are three (vector) functions in the right hand side of (2.4):

$$
\begin{gathered}
\boldsymbol{\alpha}(\mu)=\boldsymbol{p} \int_{0}^{t} e^{-\frac{1}{\mu} A(\mu, s)} d s \\
\boldsymbol{\beta}(\mu)=\frac{1}{\mu} \int_{0}^{t} e^{-\frac{1}{\mu} A(\mu, s)}\left(\int_{0}^{s} e^{\frac{1}{\mu} A(\mu, r)} \boldsymbol{b}\left(\boldsymbol{q}_{r}^{\mu}\right) d r\right) d s, \\
\gamma(\mu)=\frac{1}{\mu} \int_{0}^{t} e^{-\frac{1}{\mu} A(\mu, s)}\left(\int_{0}^{s} e^{\frac{1}{\mu} A(\mu, r)} d \boldsymbol{W}_{r}\right) d s .
\end{gathered}
$$


In the following we will use the relation

$$
\frac{d}{d t}\left(e^{-\frac{1}{\mu} A(\mu, t)}\right)=-\frac{1}{\mu} e^{-\frac{1}{\mu} A(\mu, t)} \frac{d A(\mu, t)}{d t}=-\frac{1}{\mu} e^{-\frac{1}{\mu} A(\mu, t)} \lambda\left(\boldsymbol{q}_{t}^{\mu}\right) .
$$

We will also use the estimates

$$
\begin{gathered}
\frac{\mu}{c \Lambda}\left(1-e^{-\frac{c \Lambda t}{\mu}}\right)=\int_{0}^{t} e^{-\frac{c \Lambda s}{\mu}} d s \leq \int_{0}^{t} e^{-\frac{c}{\mu} A(\mu, s)} d s \leq \int_{0}^{t} e^{-\frac{c \lambda_{0} s}{\mu}} d s=\frac{\mu}{c \lambda_{0}}\left(1-e^{-\frac{c \lambda_{0} t}{\mu}}\right) \leq \frac{\mu}{c \lambda_{0}}, \\
\frac{\mu}{c \Lambda}\left(1-e^{-\frac{c \Lambda t}{\mu}}\right)=\int_{0}^{t} e^{-\frac{c \Lambda(t-s)}{\mu}} d s \leq \int_{0}^{t} e^{-\frac{c}{\mu}(A(\mu, t)-A(\mu, s))} d s \leq \\
\leq \int_{0}^{t} e^{-\frac{c \lambda_{0}(t-s)}{\mu}} d s=\frac{\mu}{c \lambda_{0}}\left(1-e^{-\frac{c \lambda_{0} t}{\mu}}\right) \leq \frac{\mu}{c \lambda_{0}}
\end{gathered}
$$

Here $c$ is a positive constant.

We get in this section some bounds for $\boldsymbol{\alpha}(\mu), \boldsymbol{\beta}(\mu), \gamma(\mu)$ which show, in particular, that the classical Smoluchowski-Kramers approximation does not hold in the case of variable friction. These bounds also will be used to obtain a modified SmoluchowskiKramers approximation.

\subsection{Estimates of $\alpha(\mu)$.}

We have, by (2.5),

$$
\begin{aligned}
& \boldsymbol{\alpha}(\mu)=\boldsymbol{p} \int_{0}^{t} e^{-\frac{1}{\mu} A(\mu, s)} d s \\
& =\boldsymbol{p} \int_{0}^{t}(-\mu) \frac{1}{\lambda\left(\boldsymbol{q}_{s}^{\mu}\right)} d\left(e^{-\frac{1}{\mu} A(\mu, s)}\right) \\
& =-\boldsymbol{p} \mu\left[\frac{e^{-\frac{1}{\mu} A(\mu, t)}}{\lambda\left(\boldsymbol{q}_{t}^{\mu}\right)}-\frac{1}{\lambda(\boldsymbol{q})}-\int_{0}^{t} e^{-\frac{1}{\mu} A(\mu, s)} d\left(\frac{1}{\lambda\left(\boldsymbol{q}_{s}^{\mu}\right)}\right)\right] .
\end{aligned}
$$

Let $R_{\boldsymbol{\alpha}}(\mu)=\mu\left[\frac{e^{-\frac{1}{\mu} A(\mu, t)}}{\lambda\left(\boldsymbol{q}_{t}^{\mu}\right)}-\frac{1}{\lambda(\boldsymbol{q})}\right]$. It is easy to estimate $\left|R_{\boldsymbol{\alpha}}(\mu)\right| \leq \frac{\mu}{\lambda_{0}}$. Therefore $\left|R_{\boldsymbol{\alpha}}(\mu)\right| \rightarrow 0$ as $\mu \downarrow 0$.

Let

$$
(I)=\int_{0}^{t} e^{-\frac{1}{\mu} A(\mu, s)} d\left(\frac{1}{\lambda\left(\boldsymbol{q}_{s}^{\mu}\right)}\right) .
$$

We have

$$
\begin{aligned}
& (I)=-\int_{0}^{t} e^{-\frac{1}{\mu} A(\mu, s)} \frac{1}{\lambda^{2}\left(q_{s}^{\mu}\right)} \nabla \lambda\left(q_{s}^{\mu}\right) \cdot \boldsymbol{p}_{s}^{\mu} d s \\
& =-\int_{0}^{t} e^{-\frac{1}{\mu} A(\mu, s)} \frac{1}{\lambda^{2}\left(q_{s}^{\mu}\right)} e^{-\frac{1}{\mu} A(\mu, s)} \nabla \lambda\left(q_{s}^{\mu}\right) \cdot\left(\boldsymbol{p}+\frac{1}{\mu} \int_{0}^{s} e^{\frac{1}{\mu} A(\mu, r)} \boldsymbol{b}\left(\boldsymbol{q}_{r}^{\mu}\right) d r+\frac{1}{\mu} \int_{0}^{s} e^{\frac{1}{\mu} A(\mu, r)} d \boldsymbol{W}_{r}\right) d s \\
& =\left(I_{1}\right)+\left(I_{2}\right)+\left(I_{3}\right) .
\end{aligned}
$$


Here

$$
\begin{gathered}
\left(I_{1}\right)=-\boldsymbol{p} \cdot \int_{0}^{t} e^{-\frac{2}{\mu} A(\mu, s)} \frac{\nabla \lambda\left(\boldsymbol{q}_{s}^{\mu}\right)}{\lambda^{2}\left(\boldsymbol{q}_{s}^{\mu}\right)} d s \\
\left(I_{2}\right)=-\frac{1}{\mu} \int_{0}^{t} e^{-\frac{2}{\mu} A(\mu, s)} \frac{1}{\lambda^{2}\left(\boldsymbol{q}_{s}^{\mu}\right)} \nabla \lambda\left(\boldsymbol{q}_{s}^{\mu}\right) \cdot\left(\int_{0}^{s} e^{\frac{1}{\mu} A(\mu, r)} \boldsymbol{b}\left(\boldsymbol{q}_{r}^{\mu}\right) d r\right) d s, \\
\left(I_{3}\right)=-\frac{1}{\mu} \int_{0}^{t} e^{-\frac{2}{\mu} A(\mu, s)} \frac{1}{\lambda^{2}\left(q_{s}^{\mu}\right)} \nabla \lambda\left(q_{s}^{\mu}\right) \cdot\left(\int_{0}^{s} e^{\frac{1}{\mu} A(\mu, r)} d \boldsymbol{W}_{r}\right) d s .
\end{gathered}
$$

We can derive, using (2.6) and (2.7), that

$$
\begin{aligned}
& \left|\left(I_{1}\right)\right| \leq \frac{\|\nabla \lambda\|_{\infty}}{\lambda_{0}^{2}}|\boldsymbol{p}|_{\mathbb{R}^{d}} \int_{0}^{t} e^{-\frac{2}{\mu} \lambda_{0} s} d s \leq \frac{\|\nabla \lambda\|_{\infty}}{\lambda_{0}^{2}}|\boldsymbol{p}|_{\mathbb{R}^{d}} \frac{\mu}{2 \lambda_{0}} \\
& \left|\left(I_{2}\right)\right| \leq \frac{\|\nabla \lambda\|_{\infty}}{\lambda_{0}^{2}}\|\boldsymbol{b}\|_{\infty} \frac{1}{\mu} \int_{0}^{t} e^{-\frac{2}{\mu} A(\mu, s)}\left(\int_{0}^{s} e^{\frac{1}{\mu} A(\mu, r)} d r\right) d s \\
& \leq \frac{\|\nabla \lambda\|_{\infty}}{\lambda_{0}^{2}}\|\boldsymbol{b}\|_{\infty} \frac{1}{\mu} \int_{0}^{t}\left(\int_{0}^{s} e^{-\frac{1}{\mu}(s-r) \lambda_{0}} d r\right) e^{-\frac{1}{\mu} \lambda_{0} s} d s \\
& =\frac{\|\nabla \lambda\|_{\infty}}{\lambda_{0}^{2}}\|\boldsymbol{b}\|_{\infty} \frac{1}{\mu} \int_{0}^{t} \frac{\mu}{\lambda_{0}}\left(1-e^{-\frac{\lambda_{0} s}{\mu}}\right) e^{-\frac{\lambda_{0} s}{\mu}} d s \\
& \leq \frac{\|\nabla \lambda\|_{\infty}}{\lambda_{0}^{3}}\|\boldsymbol{b}\|_{\infty} \int_{0}^{t} e^{-\frac{\lambda_{0} s}{\mu}} d s \\
& \leq \frac{\|\nabla \lambda\|_{\infty}}{\lambda_{0}^{3}}\|\boldsymbol{b}\|_{\infty} \frac{\mu}{\lambda_{0}} .
\end{aligned}
$$

Since

$$
\left|\left(I_{3}\right)\right| \leq \frac{\|\nabla \lambda\|_{\infty}}{\lambda_{0}^{2}} \frac{1}{\mu}\left|\int_{0}^{t} e^{-\frac{1}{2 \mu} A(\mu, s)}\left(\int_{0}^{s} e^{-\frac{1}{2 \mu} A(\mu, s)} e^{-\frac{1}{\mu} A(\mu, s)+\frac{1}{\mu} A(\mu, r)} d \boldsymbol{W}_{r}\right) d s\right|_{\mathbb{R}^{d}}
$$

we could estimate, by Cauchy-Schwarz inequality and (2.6), (2.7), that 


$$
\begin{aligned}
& \mathbf{E}\left|\left(I_{3}\right)\right|^{2} \leq\left(\frac{\|\nabla \lambda\|_{\infty}}{\lambda_{0}^{2}}\right)^{2} \frac{1}{\mu^{2}} \mathbf{E}\left|\int_{0}^{t} e^{-\frac{1}{2 \mu} A(\mu, s)}\left(\int_{0}^{s} e^{-\frac{1}{2 \mu} A(\mu, s)} e^{-\frac{1}{\mu} A(\mu, s)+\frac{1}{\mu} A(\mu, r)} d \boldsymbol{W}_{r}\right) d s\right|_{\mathbb{R}^{d}}^{2} \\
& \leq\left(\frac{\|\nabla \lambda\|_{\infty}}{\lambda_{0}^{2}}\right)^{2} \frac{1}{\mu^{2}} \mathbf{E}\left(\int_{0}^{t} e^{-\frac{1}{\mu} A(\mu, s)} d s\right)\left(\int_{0}^{t} e^{-\frac{1}{\mu} A(\mu, s)}\left|\int_{0}^{s} e^{-\frac{1}{\mu} A(\mu, s)+\frac{1}{\mu} A(\mu, r)} d \boldsymbol{W}_{r}\right|_{\mathbb{R}^{d}}^{2} d s\right)^{2} \\
& \leq\left(\frac{\|\nabla \lambda\|_{\infty}}{\lambda_{0}^{2}}\right)^{2} \frac{1}{\mu^{2}}\left(\int_{0}^{t} e^{-\frac{\lambda_{0} s}{\mu}} d s\right)\left(\int_{0}^{t} e^{-\frac{\lambda_{0} s}{\mu}} \mathbf{E}\left|\int_{0}^{s} e^{-\frac{1}{\mu} A(\mu, s)+\frac{1}{\mu} A(\mu, r)} d \boldsymbol{W}_{r}\right|_{\mathbb{R}^{d}}^{2} d s\right)^{t} \\
& =\left(\frac{\|\nabla \lambda\|_{\infty}}{\lambda_{0}^{2}}\right)^{2} \frac{1}{\mu^{2}}\left(\int_{0}^{t} e^{-\frac{\lambda_{0} s}{\mu}} d s\right)\left(\int_{0}^{t} e^{-\frac{\lambda_{0} s}{\mu}}\left(\int_{0}^{s} \mathbf{E} e^{-\frac{2}{\mu} A(\mu, s)+\frac{2}{\mu} A(\mu, r)} d r\right) d s\right) \\
& \leq\left(\frac{\|\nabla \lambda\|_{\infty}}{\lambda_{0}^{2}}\right)^{2} \frac{1}{\mu^{2}}\left(\int_{0}^{t} e^{-\frac{\lambda_{0} s}{\mu}} d s\right)\left(\int_{0}^{t} e^{-\frac{\lambda_{0} s}{\mu}}\left(\int_{0}^{s} e^{-\frac{2 \lambda_{0} s}{\mu}+\frac{2 \lambda_{0} r}{\mu}} d r\right) d s\right) \\
& \leq\left(\frac{\|\nabla \lambda\|_{\infty}}{\lambda_{0}^{2}}\right)^{2} \frac{1}{\mu^{2}} \frac{\mu}{\lambda_{0}}\left(\int_{0}^{t} e^{-\frac{\lambda_{0} s}{\mu}} \frac{\mu}{2 \lambda_{0}} d s\right) \\
& \leq\left(\frac{\|\nabla \lambda\|_{\infty}}{\lambda_{0}^{2}}\right)^{2} \frac{\mu}{2 \lambda_{0}^{3}} .
\end{aligned}
$$

Combining these estimates we see that $\mathbf{E}|(I)|^{2} \rightarrow 0$ as $\mu \downarrow 0$.

So $\mathbf{E}|\boldsymbol{\alpha}(\mu)|_{\mathbb{R}^{d}}^{2} \rightarrow 0$ as $\mu \downarrow 0$ for any $|\boldsymbol{p}|_{\mathbb{R}^{d}}<\infty$.

\subsection{Estimates of $\boldsymbol{\beta}(\mu)$.}

We have, by (2.5), that

$$
\begin{aligned}
& \boldsymbol{\beta}(\mu)=\frac{1}{\mu} \int_{0}^{t} e^{-\frac{1}{\mu} A(\mu, s)}\left(\int_{0}^{s} e^{\frac{1}{\mu} A(\mu, r)} \boldsymbol{b}\left(\boldsymbol{q}_{r}^{\mu}\right) d r\right) d s \\
& =\frac{1}{\mu} \int_{0}^{t}\left(\int_{0}^{s} e^{\frac{1}{\mu} A(\mu, r)} \boldsymbol{b}\left(\boldsymbol{q}_{r}^{\mu}\right) d r\right)\left(-\frac{\mu}{\lambda\left(\boldsymbol{q}_{s}^{\mu}\right)}\right) d\left(e^{-\frac{1}{\mu} A(\mu, s)}\right) \\
& =\int_{0}^{t}\left(\int_{0}^{s} e^{\frac{1}{\mu} A(\mu, r)} \boldsymbol{b}\left(\boldsymbol{q}_{r}^{\mu}\right) d r\right)\left(-\frac{1}{\lambda\left(\boldsymbol{q}_{s}^{\mu}\right)}\right) d\left(e^{-\frac{1}{\mu} A(\mu, s)}\right) \\
& =-\left.\frac{e^{-\frac{1}{\mu} A(\mu, s)}}{\lambda\left(\boldsymbol{q}_{s}^{\mu}\right)} \int_{0}^{s} e^{\frac{1}{\mu} A(\mu, r)} \boldsymbol{b}\left(\boldsymbol{q}_{r}^{\mu}\right) d r\right|_{s=0} ^{s=t}+\int_{0}^{t} e^{-\frac{1}{\mu} A(\mu, s)} d\left(\int_{0}^{s} e^{\frac{1}{\mu} A(\mu, r)} \boldsymbol{b}\left(\boldsymbol{q}_{r}^{\mu}\right) d r \frac{1}{\lambda\left(\boldsymbol{q}_{s}^{\mu}\right)}\right) \\
& =-\frac{e^{-\frac{1}{\mu} A(\mu, t)}}{\lambda\left(\boldsymbol{q}_{t}^{\mu}\right)} \int_{0}^{t} e^{\frac{1}{\mu} A(\mu, s)} \boldsymbol{b}\left(\boldsymbol{q}_{s}^{\mu}\right) d s+\int_{0}^{t} \frac{\boldsymbol{b}\left(\boldsymbol{q}_{s}^{\mu}\right)}{\lambda\left(\boldsymbol{q}_{s}^{\mu}\right)} d s+\int_{0}^{t} e^{-\frac{1}{\mu} A(\mu, s)}\left(\int_{0}^{s} e^{\frac{1}{\mu} A(\mu, r)} \boldsymbol{b}\left(\boldsymbol{q}_{r}^{\mu}\right) d r\right) d\left(\frac{1}{\lambda\left(\boldsymbol{q}_{s}^{\mu}\right)}\right) \\
& =\boldsymbol{R}_{\boldsymbol{\beta}}(\mu)+\int_{0}^{t} \frac{\boldsymbol{b}\left(\boldsymbol{q}_{s}^{\mu}\right)}{\lambda\left(\boldsymbol{q}_{s}^{\mu}\right)} d s+(\overrightarrow{I I}) .
\end{aligned}
$$

It is easy to see that

$$
\left|\boldsymbol{R}_{\boldsymbol{\beta}}(\mu)\right|_{\mathbb{R}^{d}} \leq \frac{\|\boldsymbol{b}\|_{\infty}}{\lambda_{0}} \int_{0}^{t} e^{-\frac{\lambda_{0}}{\mu}(t-s)} d s=\frac{\|\boldsymbol{b}\|_{\infty}}{\lambda_{0}} \frac{\mu}{\lambda_{0}} .
$$

We also have 


$$
\begin{gathered}
(\overrightarrow{I I})=-\int_{0}^{t} e^{-\frac{1}{\mu} A(\mu, s)}\left(\int_{0}^{s} e^{\frac{1}{\mu} A(\mu, r)} \boldsymbol{b}\left(\boldsymbol{q}_{r}^{\mu}\right) d r\right) \frac{1}{\lambda^{2}\left(\boldsymbol{q}_{s}^{\mu}\right)} \nabla \lambda\left(\boldsymbol{q}_{s}^{\mu}\right) \cdot \boldsymbol{p}_{s}^{\mu} d s \\
=-\int_{0}^{t} e^{-\frac{1}{\mu} A(\mu, s)}\left(\int_{0}^{s} e^{\frac{1}{\mu} A(\mu, r)} \boldsymbol{b}\left(\boldsymbol{q}_{r}^{\mu}\right) d r\right) \frac{1}{\lambda^{2}\left(\boldsymbol{q}_{s}^{\mu}\right)} \nabla \lambda\left(\boldsymbol{q}_{s}^{\mu}\right) . \\
\cdot e^{-\frac{1}{\mu} A(\mu, s)}\left(\boldsymbol{p}+\frac{1}{\mu} \int_{0}^{s} e^{\frac{1}{\mu} A(\mu, r)} \boldsymbol{b}\left(\boldsymbol{q}_{r}^{\mu}\right) d r+\frac{1}{\mu} \int_{0}^{s} e^{\frac{1}{\mu} A(\mu, r)} d \boldsymbol{W}_{r}\right) d s \\
=\left(I \vec{I}_{1}\right)+\left(I \vec{I}_{2}\right)+\left(I \vec{I}_{3}\right) .
\end{gathered}
$$

Here

$$
\begin{gathered}
\left(I \vec{I}_{1}\right)=-\int_{0}^{t} \frac{e^{-\frac{2}{\mu} A(\mu, s)}}{\lambda^{2}\left(\boldsymbol{q}_{s}^{\mu}\right)}\left(\int_{0}^{s} e^{\frac{1}{\mu} A(\mu, r)} \boldsymbol{b}\left(\boldsymbol{q}_{r}^{\mu}\right) d r\right) \nabla \lambda\left(\boldsymbol{q}_{s}^{\mu}\right) \cdot \boldsymbol{p} d s \\
\left(I \vec{I}_{2}\right)=-\frac{1}{\mu} \int_{0}^{t} \frac{e^{-\frac{2}{\mu} A(\mu, s)}}{\lambda^{2}\left(\boldsymbol{q}_{s}^{\mu}\right)}\left(\int_{0}^{s} e^{\frac{1}{\mu} A(\mu, r)} \boldsymbol{b}\left(\boldsymbol{q}_{r}^{\mu}\right) d r\right) \nabla \lambda\left(\boldsymbol{q}_{s}^{\mu}\right) \cdot\left(\int_{0}^{s} e^{\frac{1}{\mu} A(\mu, r)} \boldsymbol{b}\left(\boldsymbol{q}_{r}^{\mu}\right) d r\right) d s \\
\left(I \vec{I}_{3}\right)=-\frac{1}{\mu} \int_{0}^{t} \frac{e^{-\frac{2}{\mu} A(\mu, s)}}{\lambda^{2}\left(\boldsymbol{q}_{s}^{\mu}\right)}\left(\int_{0}^{s} e^{\frac{1}{\mu} A(\mu, r)} \boldsymbol{b}\left(\boldsymbol{q}_{r}^{\mu}\right) d r\right) \nabla \lambda\left(\boldsymbol{q}_{s}^{\mu}\right) \cdot\left(\int_{0}^{s} e^{\frac{1}{\mu} A(\mu, r)} d \boldsymbol{W}_{r}\right) d s .
\end{gathered}
$$

We conclude that

$$
\begin{aligned}
& \left|\left(I \vec{I}_{1}\right)\right|_{\mathbb{R}^{d}} \leq \frac{\|\nabla \lambda\|_{\infty}}{\lambda_{0}^{2}}|\boldsymbol{p}|_{\mathbb{R}^{d}}\|\boldsymbol{b}\|_{\infty} \int_{0}^{t} e^{-\frac{\lambda_{0} s}{\mu}}\left(\int_{0}^{s} e^{-\frac{(s-r) \lambda_{0}}{\mu}} d r\right) d s \\
& \leq \frac{\|\nabla \lambda\|_{\infty}}{\lambda_{0}^{2}}|\boldsymbol{p}|_{\mathbb{R}^{d}}\|\boldsymbol{b}\|_{\infty} \frac{\mu^{2}}{\lambda_{0}^{2}} ; \\
& \left|\left(I \vec{I}_{2}\right)\right|_{\mathbb{R}^{d}} \leq \frac{1}{\mu} \frac{\|\nabla \lambda\|_{\infty}}{\lambda_{0}^{2}}\|\boldsymbol{b}\|_{\infty}^{2} \int_{0}^{t}\left(\int_{0}^{s} e^{-\frac{(s-r) \lambda_{0}}{\mu}} d r\right)^{2} d s \\
& \leq \frac{\|\nabla \lambda\|_{\infty}}{\lambda_{0}^{2}}\|\boldsymbol{b}\|_{\infty}^{2} \frac{\mu t}{\lambda_{0}^{2}}
\end{aligned}
$$

$\mathbf{E}\left|\left(I \vec{I}_{3}\right)\right|_{\mathbb{R}^{d}}^{2} \leq\left(\frac{1}{\mu} \frac{\|\nabla \lambda\|_{\infty}}{\lambda_{0}^{2}}\|\boldsymbol{b}\|_{\infty}\right)^{2} \mathbf{E}\left|\int_{0}^{t} e^{-\frac{2}{\mu} A(\mu, s)}\left(\int_{0}^{s} e^{\frac{1}{\mu} A(\mu, r)} d r\right)\left(\int_{0}^{s} e^{\frac{1}{\mu} A(\mu, r)} d \boldsymbol{W}_{r}\right) d s\right|_{\mathbb{R}^{d}}^{2}$

$=\left(\frac{1}{\mu} \frac{\|\nabla \lambda\|_{\infty}}{\lambda_{0}^{2}}\|\boldsymbol{b}\|_{\infty}\right)^{2} \mathbf{E}\left|\int_{0}^{t}\left(\int_{0}^{s} e^{-\frac{1}{\mu}(A(\mu, s)-A(\mu, r))} d r\right)\left(\int_{0}^{s} e^{-\frac{1}{\mu}(A(\mu, s)-A(\mu, r))} d \boldsymbol{W}_{r}\right) d s\right|_{\mathbb{R}^{d}}^{2^{\mathbb{R}^{d}}}$

$\leq\left(\frac{1}{\mu} \frac{\|\nabla \lambda\|_{\infty}}{\lambda_{0}^{2}}\|\boldsymbol{b}\|_{\infty}\right)^{2} \mathbf{E}\left(\int_{0}^{t}\left(\int_{0}^{s} e^{-\frac{1}{\mu}(A(\mu, s)-A(\mu, r))} d r\right)^{2} d s\right)\left(\int_{0}^{t}\left|\int_{0}^{s} e^{-\frac{1}{\mu}(A(\mu, s)-A(\mu, r))} d \boldsymbol{W}_{r}\right|_{\mathbb{R}^{d}}^{2} d s\right)$

$\leq\left(\frac{1}{\mu} \frac{\|\nabla \lambda\|_{\infty}}{\lambda_{0}^{2}}\|\boldsymbol{b}\|_{\infty}\right)^{2}\left(\int_{0}^{t}\left(\int_{0}^{s} e^{-\frac{(s-r) \lambda_{0}}{\mu}} d r\right)^{2} d s\right)\left(\int_{0}^{t} \mathbf{E}\left|\int_{0}^{s} e^{-\frac{1}{\mu}(A(\mu, s)-A(\mu, r))} d \boldsymbol{W}_{r}\right|_{\mathbb{R}^{d}}^{2} d s\right)$

$\leq\left(\frac{1}{\mu} \frac{\|\nabla \lambda\|_{\infty}}{\lambda_{0}^{2}}\|\boldsymbol{b}\|_{\infty}\right)^{2}\left(\int_{0}^{t}\left(\int_{0}^{s} e^{-\frac{(s-r) \lambda_{0}}{\mu}} d r\right)^{2} d s\right)\left(\int_{0}^{t}\left(\int_{0}^{s} e^{-\frac{2(s-r) \lambda_{0}}{\mu}} d r\right) d s\right)$

$\leq\left(\frac{\|\nabla \lambda\|_{\infty}}{\lambda_{0}^{2}}\|\boldsymbol{b}\|_{\infty}\right)^{2}\left(\frac{t}{\lambda_{0}}\right)^{2}\left(\frac{\mu t}{2 \lambda_{0}}\right)$. 
Combining these estimates we see that $\mathbf{E}|(\overrightarrow{I I})|_{\mathbb{R}^{d}}^{2} \rightarrow 0$ as $\mu \downarrow 0$. This implies that $\mathbf{E}\left|\boldsymbol{\beta}(\mu)-\int_{0}^{t} \frac{\boldsymbol{b}\left(\boldsymbol{q}_{s}^{\mu}\right)}{\lambda\left(\boldsymbol{q}_{s}^{\mu}\right)} d s\right|_{\mathbb{R}^{d}}^{2} \rightarrow 0$ as $\mu \downarrow 0$.

2.3. Estimates of $\gamma(\mu)$ - the reason why the classical SmoluchowskiKramers approximation does not work.

We will show that $\mathbf{E}\left|\gamma(\mu)-\int_{0}^{t} \frac{1}{\lambda\left(\boldsymbol{q}_{s}^{\mu}\right)} d \boldsymbol{W}_{s}\right|_{\mathbb{R}^{d}}^{2}$, in general, does not tend to 0 as $\mu \downarrow 0$. Therefore the Smoluchowski-Kramers approximation does not work in the case of purely white noise perturbation.

We have, by (2.5), that

$$
\begin{aligned}
& \gamma(\mu)=\frac{1}{\mu} \int_{0}^{t} e^{-\frac{1}{\mu} A(\mu, s)}\left(\int_{0}^{s} e^{\frac{1}{\mu} A(\mu, r)} d \boldsymbol{W}_{r}\right) d s \\
& =\frac{1}{\mu} \int_{0}^{t}\left(\int_{0}^{s} e^{\frac{1}{\mu} A(\mu, r)} d \boldsymbol{W}_{r}\right)\left(-\frac{\mu}{\lambda\left(\boldsymbol{q}_{s}^{\mu}\right)}\right) d\left(e^{-\frac{1}{\mu} A(\mu, s)}\right) \\
& =-\left[\frac{\int_{0}^{t} e^{\frac{1}{\mu} A(\mu, s)} d \boldsymbol{W}_{s}}{\lambda\left(\boldsymbol{q}_{t}^{\mu}\right)} e^{-\frac{1}{\mu} A(\mu, t)}-\int_{0}^{t} e^{-\frac{1}{\mu} A(\mu, s)} d\left(\frac{1}{\lambda\left(\boldsymbol{q}_{s}^{\mu}\right)} \int_{0}^{s} e^{\frac{1}{\mu} A(\mu, r)} d \boldsymbol{W}_{r}\right)\right] \\
& =-\frac{\int_{0}^{t} e^{\frac{1}{\mu} A(\mu, s)} d \boldsymbol{W}_{s}}{\lambda\left(\boldsymbol{q}_{t}^{\mu}\right)} e^{-\frac{1}{\mu} A(\mu, t)}+\int_{0}^{t} \frac{1}{\lambda\left(\boldsymbol{q}_{s}^{\mu}\right)} d \boldsymbol{W}_{s}+ \\
& \quad+\int_{0}^{t} e^{-\frac{1}{\mu} A(\mu, s)}\left(\int_{0}^{s} e^{\frac{1}{\mu} A(\mu, r)} d \boldsymbol{W}_{r}\right) d\left(\frac{1}{\lambda\left(\boldsymbol{q}_{s}^{\mu}\right)}\right) \\
& =\boldsymbol{R}_{\gamma}(\mu)+\int_{0}^{t} \frac{1}{\lambda\left(\boldsymbol{q}_{s}^{\mu}\right)} d \boldsymbol{W}_{s}+(I \vec{I} I) .
\end{aligned}
$$

It is easy to check that

$$
\mathbf{E}\left|\boldsymbol{R}_{\gamma}(\mu)\right|_{\mathbb{R}^{d}}^{2} \leq \frac{1}{\lambda_{0}^{2}} \int_{0}^{t} e^{-\frac{2 \lambda_{0}(t-s)}{\mu}} d s \leq \frac{\mu}{2 \lambda_{0}^{3}}
$$

We have

$$
\begin{aligned}
& (I \vec{I} I)=\int_{0}^{t} e^{-\frac{1}{\mu} A(\mu, s)}\left(\int_{0}^{s} e^{\frac{1}{\mu} A(\mu, r)} d \boldsymbol{W}_{r}\right)\left(-\frac{1}{\lambda^{2}\left(\boldsymbol{q}_{s}^{\mu}\right)}\right) \nabla \lambda\left(\boldsymbol{q}_{s}^{\mu}\right) \cdot \boldsymbol{p}_{s}^{\mu} d s \\
& =\left(I \overrightarrow{I I}_{1}\right)+\left(I \overrightarrow{I I}_{2}\right)+\left(I \overrightarrow{I I}_{3}\right)
\end{aligned}
$$

where

$$
\left(I \overrightarrow{I I}_{1}\right)=-\int_{0}^{t} \frac{e^{-\frac{2}{\mu} A(\mu, s)}}{\lambda^{2}\left(\boldsymbol{q}_{s}^{\mu}\right)}\left(\int_{0}^{s} e^{\frac{1}{\mu} A(\mu, r)} d \boldsymbol{W}_{r}\right) \nabla \lambda\left(\boldsymbol{q}_{s}^{\mu}\right) \cdot \boldsymbol{p} d s
$$




$$
\begin{gathered}
\left(I \overrightarrow{I I}_{2}\right)=-\frac{1}{\mu} \int_{0}^{t} \frac{e^{-\frac{2}{\mu} A(\mu, s)}}{\lambda^{2}\left(\boldsymbol{q}_{s}^{\mu}\right)}\left(\int_{0}^{s} e^{\frac{1}{\mu} A(\mu, r)} d \boldsymbol{W}_{r}\right) \nabla \lambda\left(\boldsymbol{q}_{s}^{\mu}\right) \cdot\left(\int_{0}^{s} e^{\frac{1}{\mu} A(\mu, r)} \boldsymbol{b}\left(\boldsymbol{q}_{r}^{\mu}\right) d r\right) d s, \\
\left(I \overrightarrow{I I}_{3}\right)=-\frac{1}{\mu} \int_{0}^{t} \frac{e^{-\frac{2}{\mu} A(\mu, s)}}{\lambda^{2}\left(\boldsymbol{q}_{s}^{\mu}\right)}\left(\int_{0}^{s} e^{\frac{1}{\mu} A(\mu, r)} d \boldsymbol{W}_{r}\right) \nabla \lambda\left(\boldsymbol{q}_{s}^{\mu}\right) \cdot\left(\int_{0}^{s} e^{\frac{1}{\mu} A(\mu, r)} d \boldsymbol{W}_{r}\right) d s,
\end{gathered}
$$

We can estimate

$$
\begin{aligned}
& \mathbf{E}\left|\left(I \overrightarrow{I I}_{1}\right)\right|_{\mathbb{R}^{d}}^{2} \leq\left(\frac{|\boldsymbol{p}|_{\mathbb{R}^{d}}\|\nabla \lambda\|_{\infty}}{\lambda_{0}^{2}}\right)^{2} \mathbf{E}\left|\int_{0}^{t} e^{-\frac{1}{\mu} A(\mu, s)}\left(\int_{0}^{s} e^{-\frac{1}{\mu}(A(\mu, s)-A(\mu, r))} d \boldsymbol{W}_{r}\right) d s\right|_{\mathbb{R}^{d}}^{2} \\
& \leq\left(\frac{|\boldsymbol{p}|_{\mathbb{R}^{d}}\|\nabla \lambda\|_{\infty}}{\lambda_{0}^{2}}\right)^{2} \mathbf{E}\left(\int_{0}^{t} e^{-\frac{2}{\mu} A(\mu, s)} d s\right)\left(\int_{0}^{t}\left|\int_{0}^{s} e^{-\frac{1}{\mu}(A(\mu, s)-A(\mu, r))} d \boldsymbol{W}_{r}\right|_{\mathbb{R}^{d}}^{2} d s\right)^{2} \\
& \leq\left(\frac{|\boldsymbol{p}|_{\mathbb{R}^{d}}\|\nabla \lambda\|_{\infty}}{\lambda_{0}^{2}}\right)^{2}\left(\int_{0}^{t} e^{-\frac{2 \lambda_{0} s}{\mu}} d s\right)\left(\int_{0}^{t}\left(\int_{0}^{s} e^{-\frac{2 \lambda_{0}(s-r)}{\mu}} d r\right) d s\right) \\
& \leq\left(\frac{|\boldsymbol{p}|_{\mathbb{R}^{d}}\|\nabla \lambda\|_{\infty}}{\lambda_{0}^{2}}\right)^{2}\left(\frac{\mu}{2 \lambda_{0}}\right)\left(\frac{\mu t}{2 \lambda_{0}}\right) .
\end{aligned}
$$

The term $\left(I \overrightarrow{I I}_{2}\right)$ could be estimated in the same way as $\left(I \vec{I}_{3}\right)$ :

$$
\mathbf{E}\left|\left(I \overrightarrow{I I}_{2}\right)\right|_{\mathbb{R}^{d}}^{2} \leq\left(\frac{\|\nabla \lambda\|_{\infty}}{\lambda_{0}^{2}}\|\boldsymbol{b}\|_{\infty}\right)^{2}\left(\frac{t}{\lambda_{0}}\right)^{2}\left(\frac{\mu t}{2 \lambda_{0}}\right) .
$$

But in general one cannot estimate $\mathbf{E}\left|\left(I_{I I}\right)\right|^{2}$ up to a term which goes to 0 as $\mu \downarrow 0$. As an example, let $\Lambda=\|\lambda\|_{\infty}$ and let us suppose that for $0 \leq t \leq T<\infty$ we have $\nabla \lambda\left(\boldsymbol{q}_{t}^{\mu}\right)=\mathbf{e}_{1}$. Here $\mathbf{e}_{1}$ is the unit basis vector $\mathbf{e}_{1}=(1,0, \ldots, 0)$ in $\mathbb{R}^{d}$. Let $W_{r}^{k}$ be the $k$-th $(1 \leq k \leq d)$ component of the Wiener process $\boldsymbol{W}_{r}$. We have, for $0<t \leq T$ :

$$
\begin{aligned}
& \mathbf{E}\left|\left(I \overrightarrow{I I} I_{3}\right)\right|_{\mathbb{R}^{d}} \geq \frac{1}{\mu \Lambda^{2}} \mathbf{E}\left|\int_{0}^{t}\left(\int_{0}^{s} e^{-\frac{1}{\mu}(A(\mu, s)-A(\mu, r))} d \boldsymbol{W}_{r}\right)\left(\int_{0}^{s} e^{-\frac{1}{\mu}(A(\mu, s)-A(\mu, r))} d W_{r}^{1}\right) d s\right|_{\mathbb{R}^{d}} \\
& =\frac{1}{\mu \Lambda^{2}} \mathbf{E}\left[\left(\int_{0}^{t}\left(\int_{0}^{s} e^{-\frac{1}{\mu}(A(\mu, s)-A(\mu, r))} d W_{r}^{1}\right)^{2} d s\right)^{2}+\right. \\
& \left.\quad+\sum_{k=2}^{d}\left(\int_{0}^{t}\left(\int_{0}^{s} e^{-\frac{1}{\mu}(A(\mu, s)-A(\mu, r))} d W_{r}^{k}\right)\left(\int_{0}^{s} e^{-\frac{1}{\mu}(A(\mu, s)-A(\mu, r))} d W_{r}^{1}\right) d s\right)^{2}\right]^{\frac{1}{2}} \\
& \geq \frac{1}{\mu \Lambda^{2}} \mathbf{E}\left(\int_{0}^{t}\left(\int_{0}^{s} e^{-\frac{1}{\mu}(A(\mu, s)-A(\mu, r))} d W_{r}^{1}\right)^{2} d s\right) \\
& =\frac{1}{\mu \Lambda^{2}}\left(\int_{0}^{t}\left(\int_{0}^{s} \mathbf{E} e^{-\frac{2}{\mu}(A(\mu, s)-A(\mu, r))} d r\right) d s\right) \\
& \geq \frac{1}{\mu \Lambda^{2}}\left(\int_{0}^{t}\left(\int_{0}^{s} e^{-\frac{2}{\mu} \Lambda(s-r)} d r\right) d s\right) \\
& =\frac{1}{\mu \Lambda^{2}} \frac{\mu}{2 \Lambda} \int_{0}^{t}\left(1-e^{-\frac{2 \Lambda s}{\mu}}\right) d s=\frac{t}{2 \Lambda^{3}}-\frac{\mu}{4 \Lambda^{4}}\left(1-e^{-\frac{2 \Lambda t}{\mu}}\right),
\end{aligned}
$$


which does not tend to 0 as $\mu \downarrow 0$. Since $\mathbf{E}\left|\left(I \overrightarrow{I I} I_{3}\right)\right|_{\mathbb{R}^{d}}^{2} \geq\left(\mathbf{E}\left|\left(I \overrightarrow{I I}_{3}\right)\right|_{\mathbb{R}^{d}}\right)^{2}$, we see that $\mathbf{E}\left|\left(I \overrightarrow{I I}_{3}\right)\right|_{\mathbb{R}^{d}}^{2}$ does not go to 0 as $\mu \downarrow 0$. Now we have

$\left.\mathbf{E}\left|\gamma(\mu)-\int_{0}^{t} \frac{1}{\lambda\left(\boldsymbol{q}_{s}^{\mu}\right)} d \boldsymbol{W}_{s}\right|_{\mathbb{R}^{d}}^{2} \geq \frac{1}{4} \mathbf{E} \mid(I \overrightarrow{I I})_{3}\right)\left.\right|_{\mathbb{R}^{d}} ^{2}-\mathbf{E}\left|\boldsymbol{R}_{\gamma}(\mu)\right|_{\mathbb{R}^{d}}^{2}-\mathbf{E}\left|\left(I \overrightarrow{I I}_{1}\right)\right|_{\mathbb{R}^{d}}^{2}-\mathbf{E}\left|\left(I \overrightarrow{I I}_{2}\right)\right|_{\mathbb{R}^{d}}^{2}$

Therefore $\mathbf{E}\left|\gamma(\mu)-\int_{0}^{t} \frac{1}{\lambda\left(\boldsymbol{q}_{s}^{\mu}\right)} d \boldsymbol{W}_{s}\right|_{\mathbb{R}^{d}}^{2}$ is uniformly bounded from below by a positive constant as $\mu \downarrow 0$.

We can check now that the process $\boldsymbol{q}_{t}^{\mu}, 0 \leq t \leq T$, does not converge as $\mu \downarrow 0$ to the process $\boldsymbol{q}_{t}, \boldsymbol{q}_{0}=\boldsymbol{q}$. We have

$$
\begin{aligned}
& \boldsymbol{q}_{t}^{\mu}=\boldsymbol{q}+\int_{0}^{t} \frac{\boldsymbol{b}\left(\boldsymbol{q}_{s}^{\mu}\right)}{\lambda\left(\boldsymbol{q}_{s}^{\mu}\right)} d s+\int_{0}^{t} \frac{1}{\lambda\left(\boldsymbol{q}_{s}^{\mu}\right)} d \boldsymbol{W}_{s}+ \\
&+\boldsymbol{\alpha}(\mu)+\left(\boldsymbol{\beta}(\mu)-\int_{0}^{t} \frac{\boldsymbol{b}\left(\boldsymbol{q}_{s}^{\mu}\right)}{\lambda\left(\boldsymbol{q}_{s}^{\mu}\right)} d s\right)+\left(\gamma(\mu)-\int_{0}^{t} \frac{1}{\lambda\left(\boldsymbol{q}_{s}^{\mu}\right)} d \boldsymbol{W}_{s}\right) \\
& \boldsymbol{q}_{t}=\boldsymbol{q}+\int_{0}^{t} \frac{\boldsymbol{b}\left(\boldsymbol{q}_{s}\right)}{\lambda\left(\boldsymbol{q}_{s}\right)} d s+\int_{0}^{t} \frac{1}{\lambda\left(\boldsymbol{q}_{s}\right)} d \boldsymbol{W}_{s} .
\end{aligned}
$$

Suppose that we have, for any $\kappa, T>0$ and any $\boldsymbol{p}_{0}^{\mu}=\boldsymbol{p} \in \mathbb{R}^{d}$ fixed, that

$$
\lim _{\mu \downarrow 0} \mathbf{P}\left(\max _{0 \leq t \leq T}\left|\boldsymbol{q}_{t}^{\mu}-\boldsymbol{q}_{t}\right|_{\mathbb{R}^{d}}^{2} \geq \kappa\right)=0
$$

We have, for some $A>0$ independent of $\mu$ and $\kappa$, that

$$
\begin{aligned}
& \mathbf{E}\left|\left(\boldsymbol{q}_{t}^{\mu}-\boldsymbol{q}_{t}\right)-\int_{0}^{t}\left(\frac{\boldsymbol{b}\left(\boldsymbol{q}_{s}^{\mu}\right)}{\lambda\left(\boldsymbol{q}_{s}^{\mu}\right)}-\frac{\boldsymbol{b}\left(\boldsymbol{q}_{s}\right)}{\lambda\left(\boldsymbol{q}_{s}\right)}\right) d s-\int_{0}^{t}\left(\frac{1}{\lambda\left(\boldsymbol{q}_{s}^{\mu}\right)}-\frac{1}{\lambda\left(\boldsymbol{q}_{s}\right)}\right) d \boldsymbol{W}_{s}\right|_{\mathbb{R}^{d}}^{2} \\
& \leq A \mathbf{E} \max _{0 \leq s \leq t}\left|\boldsymbol{q}_{s}^{\mu}-\boldsymbol{q}_{s}\right|_{\mathbb{R}^{d}}^{2} \\
& \leq A\left[\mathbf{P}\left(\max _{0 \leq s \leq t}\left|\boldsymbol{q}_{s}^{\mu}-\boldsymbol{q}_{s}\right|_{\mathbb{R}^{d}}^{2} \geq \kappa\right) \cdot \mathbf{E} \max _{0 \leq s \leq t}\left|\boldsymbol{q}_{s}^{\mu}-\boldsymbol{q}_{s}\right|_{\mathbb{R}^{d}}^{2}+\mathbf{P}\left(\max _{0 \leq s \leq t}\left|\boldsymbol{q}_{s}^{\mu}-\boldsymbol{q}_{s}\right|_{\mathbb{R}^{d}}^{2}<\kappa\right) \cdot \kappa\right] \\
& \leq A[\kappa+o(\mu, \kappa)],
\end{aligned}
$$

since $\mathbf{E} \max _{0 \leq s \leq t}\left|\boldsymbol{q}_{s}^{\mu}-\boldsymbol{q}_{s}\right|_{\mathbb{R}^{d}}^{2}<\infty$. Here the term $o(\mu, \kappa)$ converges to 0 as $\mu \downarrow 0$ for every fixed $\kappa>0$. Fix $\kappa>0$, let $\mu \downarrow 0$, we see that

$$
\lim _{\mu \downarrow 0} \mathbf{E}\left|\left(\boldsymbol{q}_{t}^{\mu}-\boldsymbol{q}_{t}\right)-\int_{0}^{t}\left(\frac{\boldsymbol{b}\left(\boldsymbol{q}_{s}^{\mu}\right)}{\lambda\left(\boldsymbol{q}_{s}^{\mu}\right)}-\frac{\boldsymbol{b}\left(\boldsymbol{q}_{s}\right)}{\lambda\left(\boldsymbol{q}_{s}\right)}\right) d s-\int_{0}^{t}\left(\frac{1}{\lambda\left(\boldsymbol{q}_{s}^{\mu}\right)}-\frac{1}{\lambda\left(\boldsymbol{q}_{s}\right)}\right) d \boldsymbol{W}_{s}\right|_{\mathbb{R}^{d}}^{2} \leq A \kappa
$$

Since $\kappa>0$ is arbitrary, we see that 


$$
\lim _{\mu \downarrow 0} \mathbf{E}\left|\left(\boldsymbol{q}_{t}^{\mu}-\boldsymbol{q}_{t}\right)-\int_{0}^{t}\left(\frac{\boldsymbol{b}\left(\boldsymbol{q}_{s}^{\mu}\right)}{\lambda\left(\boldsymbol{q}_{s}^{\mu}\right)}-\frac{\boldsymbol{b}\left(\boldsymbol{q}_{s}\right)}{\lambda\left(\boldsymbol{q}_{s}\right)}\right) d s-\int_{0}^{t}\left(\frac{1}{\lambda\left(\boldsymbol{q}_{s}^{\mu}\right)}-\frac{1}{\lambda\left(\boldsymbol{q}_{s}\right)}\right) d \boldsymbol{W}_{s}\right|_{\mathbb{R}^{d}}^{2}=0 .
$$

On the other hand, let us suppose that $\nabla \lambda\left(\boldsymbol{q}_{t}^{\mu}\right)=\mathbf{e}_{1}$ for $0 \leq t \leq T<\infty$. Here $\mathbf{e}_{1}$ is the unit basis vector $\mathbf{e}_{1}=(1,0, \ldots, 0)$ in $\mathbb{R}^{d}$. We have

$$
\begin{aligned}
& \mathbf{E}\left|\boldsymbol{\alpha}(\mu)+\left(\boldsymbol{\beta}(\mu)-\int_{0}^{t} \frac{\boldsymbol{b}\left(\boldsymbol{q}_{s}^{\mu}\right)}{\lambda\left(\boldsymbol{q}_{s}^{\mu}\right)} d s\right)+\left(\gamma(\mu)-\int_{0}^{t} \frac{1}{\lambda\left(\boldsymbol{q}_{s}^{\mu}\right)} d \boldsymbol{W}_{s}\right)\right|_{\mathbb{R}^{d}}^{2} \\
& \geq \frac{1}{3} \mathbf{E}\left|\gamma(\mu)-\int_{0}^{t} \frac{1}{\lambda\left(\boldsymbol{q}_{s}^{\mu}\right)} d \boldsymbol{W}_{s}\right|_{\mathbb{R}^{d}}^{2}-\mathbf{E}|\boldsymbol{\alpha}(\mu)|_{\mathbb{R}^{d}}^{2}-\mathbf{E}\left|\boldsymbol{\beta}(\mu)-\int_{0}^{t} \frac{\boldsymbol{b}\left(\boldsymbol{q}_{s}^{\mu}\right)}{\lambda\left(\boldsymbol{q}_{s}^{\mu}\right)} d s\right|_{\mathbb{R}^{d}}^{2} .
\end{aligned}
$$

It follows from our estimates that this leads to a contradiction.

\section{Regularization via approximation of the Wiener process}

We could regularize the problem via approximation of the Wiener process. To this end we introduce the process

$$
\boldsymbol{W}_{t}^{\delta}=\frac{1}{\delta} \int_{0}^{\infty} \boldsymbol{W}_{s} \rho\left(\frac{s-t}{\delta}\right) d s=\frac{1}{\delta} \int_{0}^{\delta} \boldsymbol{W}_{s+t} \rho\left(\frac{s}{\delta}\right) d s,
$$

where $\rho(\bullet)$ is a smooth $C^{\infty}$ function whose support is contained in the interval $[0,1]$ such that

$$
\int_{0}^{1} \rho(s) d s=1
$$

One can prove that (see [3] and the references there)

$$
\lim _{\delta \downarrow 0} \mathbf{E} \max _{t \in[0, T]}\left|\boldsymbol{W}_{t}^{\delta}-\boldsymbol{W}_{t}\right|_{\mathbb{R}^{d}}^{2}=0
$$

We have

$$
\dot{\boldsymbol{W}}_{t}^{\delta}=-\frac{1}{\delta} \int_{0}^{1} \boldsymbol{W}_{t+\delta r} \dot{\rho}(r) d r .
$$

We can then introduce the following regularization of our problem: first we consider the system

$$
\mu \ddot{\boldsymbol{q}}_{t}^{\mu, \delta}=\boldsymbol{b}\left(\boldsymbol{q}_{t}^{\mu, \delta}\right)-\lambda\left(\boldsymbol{q}_{t}^{\mu, \delta}\right) \dot{\boldsymbol{q}}_{t}^{\mu, \delta}+\dot{\boldsymbol{W}}_{t}^{\delta}, \boldsymbol{q}_{0}^{\mu, \delta}=\boldsymbol{q} \in \mathbb{R}^{d}, \dot{\boldsymbol{q}}_{0}^{\mu, \delta}=\boldsymbol{p} \in \mathbb{R}^{d}
$$

Equivalently it is the first order system 


$$
\left\{\begin{array}{l}
\dot{\boldsymbol{q}}_{t}^{\mu, \delta}=\boldsymbol{p}_{t}^{\mu, \delta} \\
\dot{\boldsymbol{p}}_{t}^{\mu, \delta}=\frac{1}{\mu} \boldsymbol{b}\left(\boldsymbol{q}_{t}^{\mu, \delta}\right)-\frac{\lambda\left(\boldsymbol{q}_{t}^{\mu, \delta}\right)}{\mu} \boldsymbol{p}_{t}^{\mu, \delta}+\frac{1}{\mu} \dot{\boldsymbol{W}}_{t}^{\delta} .
\end{array}\right.
$$

We can proceed with the estimates similar to in the previous sections. Since for fixed $\delta>0$,

$$
\left|\dot{\boldsymbol{W}}_{t}^{\delta}\right|_{\mathbb{R}^{d}} \leq \frac{1}{\delta}\left(\max _{0 \leq r \leq 1}|\dot{\rho}(r)|\right)\left(\max _{t \leq s \leq t+\delta}\left|\boldsymbol{W}_{s}\right|_{\mathbb{R}^{d}}\right)<\infty, \text { a. s. },
$$

we could prove that all the terms

$$
\mathbf{E}|\boldsymbol{\alpha}(\mu)|_{\mathbb{R}^{d}}, \mathbf{E}\left|\boldsymbol{\beta}(\mu)-\int_{0}^{t} \frac{\boldsymbol{b}\left(\boldsymbol{q}_{s}^{\mu}\right)}{\lambda\left(\boldsymbol{q}_{s}^{\mu}\right)} d s\right|_{\mathbb{R}^{d}}, \mathbf{E}\left|\gamma(\mu)-\int_{0}^{t} \frac{1}{\lambda\left(\boldsymbol{q}_{s}^{\mu}\right)} d \boldsymbol{W}_{s}^{\delta}\right|_{\mathbb{R}^{d}}
$$

goes to zero as $\mu \downarrow 0$. (To be precise, we should write $\boldsymbol{\alpha}(\mu, \delta), \boldsymbol{\beta}(\mu, \delta)$ and $\gamma(\mu, \delta)$ to indicate the dependence on $\delta$, but for brevity we neglect that.) In particular, with $\delta>0$ fixed, we can estimate the term $\left(I \overrightarrow{I I}_{3}\right)$ up to a term which tends to 0 as $\mu \downarrow 0$. We have

$$
\begin{aligned}
& \mathbf{E}\left|\left(I \overrightarrow{I I} I_{3}\right)\right|_{\mathbb{R}^{d}} \leq \frac{1}{\mu} \frac{\|\nabla \lambda\|_{\infty}}{\lambda_{0}^{2}} \int_{0}^{t} \mathbf{E}\left|\int_{0}^{s} e^{-\frac{1}{\mu}(A(\mu, s)-A(\mu, r))} \dot{\boldsymbol{W}}_{r}^{\delta} d r\right|_{\mathbb{R}^{d}}^{2} d s \\
& =\frac{1}{\mu} \frac{\|\nabla \lambda\|_{\infty}}{\lambda_{0}^{2}} \int_{0}^{t} \frac{1}{\delta^{2}} \mathbf{E}\left|\int_{0}^{s} e^{-\frac{1}{\mu}(A(\mu, s)-A(\mu, r))}\left(\int_{0}^{1} \boldsymbol{W}_{r+\delta m} \dot{\rho}(m) d m\right) d r\right|_{\mathbb{R}^{d}}^{2} d s \\
& =\frac{1}{\mu} \frac{\|\nabla \lambda\|_{\infty}}{\lambda_{0}^{2}} \int_{0}^{t} \frac{1}{\delta^{2}} \mathbf{E}\left|\int_{0}^{1} \dot{\rho}(m) \boldsymbol{W}_{r+\delta m} d m \int_{0}^{s} e^{-\frac{1}{\mu}(A(\mu, s)-A(\mu, r))} d r\right|_{\mathbb{R}^{d}}^{2} d s \\
& \leq \frac{1}{\mu} \frac{\|\nabla \lambda\|_{\infty}}{\lambda_{0}^{2}} \int_{0}^{t} \frac{1}{\delta^{2}}\left(\max _{0 \leq m \leq 1}|\dot{\rho}(m)|\right)^{2} \mathbf{E}\left(\max _{0 \leq l \leq s+\delta}\left|\boldsymbol{W}_{l}\right|_{\mathbb{R}^{d}}\right)^{2}\left(\int_{0}^{s} e^{-\frac{\lambda_{0}(s-r)}{\mu}} d r\right)^{2} d s \\
& \leq \mu \frac{\|\nabla \lambda\|_{\infty}}{\lambda_{0}^{4}} \frac{t}{\delta^{2}}\left(\max _{0 \leq m \leq 1}|\dot{\rho}(m)|\right)^{2} \mathbf{E}\left(\max _{0 \leq l \leq s+\delta}\left|\boldsymbol{W}_{l}\right|_{\mathbb{R}^{d}}\right)^{2} .
\end{aligned}
$$

Therefore, for fixed $\delta>0$, we have $\mathbf{E}\left|\left(I \overrightarrow{I I}_{3}\right)\right|_{\mathbb{R}^{d}} \rightarrow 0$ as $\mu \downarrow 0$. By (2.4), we get:

$$
\begin{aligned}
\boldsymbol{q}_{t}^{\mu, \delta}=\boldsymbol{q}+\int_{0}^{t} & \frac{\boldsymbol{b}\left(\boldsymbol{q}_{s}^{\mu, \delta}\right)}{\lambda\left(\boldsymbol{q}_{s}^{\mu, \delta}\right)} d s+\int_{0}^{t} \frac{1}{\lambda\left(\boldsymbol{q}_{s}^{\mu, \delta}\right)} d \boldsymbol{W}_{s}^{\delta}+ \\
& +\boldsymbol{\alpha}(\mu)+\left(\boldsymbol{\beta}(\mu)-\int_{0}^{t} \frac{\boldsymbol{b}\left(\boldsymbol{q}_{s}^{\mu, \delta}\right)}{\lambda\left(\boldsymbol{q}_{s}^{\mu, \delta}\right)} d s\right)+\left(\gamma(\mu)-\int_{0}^{t} \frac{1}{\lambda\left(\boldsymbol{q}_{s}^{\mu, \delta}\right)} d \boldsymbol{W}_{s}^{\delta}\right) .
\end{aligned}
$$

Let the process $\widetilde{\boldsymbol{q}}_{t}^{\delta}$ be governed by the equation

$$
\dot{\overrightarrow{\boldsymbol{q}}}_{t}^{\delta}=\frac{\boldsymbol{b}\left(\widetilde{\boldsymbol{q}}_{t}^{\delta}\right)}{\lambda\left(\widetilde{\boldsymbol{q}}_{t}^{\delta}\right)}+\frac{1}{\lambda\left(\widetilde{\boldsymbol{q}}_{t}^{\delta}\right)} \dot{\boldsymbol{W}}_{t}^{\delta}, \widetilde{\boldsymbol{q}}_{0}^{\delta}=\boldsymbol{q} \in \mathbb{R}^{d}
$$

Then 


$$
\tilde{\boldsymbol{q}}_{t}^{\delta}=\boldsymbol{q}+\int_{0}^{t} \frac{\boldsymbol{b}\left(\tilde{\boldsymbol{q}}_{s}^{\delta}\right)}{\lambda\left(\tilde{\boldsymbol{q}}_{s}^{\delta}\right)} d s+\int_{0}^{t} \frac{1}{\lambda\left(\tilde{\boldsymbol{q}}_{s}^{\delta}\right)} d \boldsymbol{W}_{s}^{\delta} .
$$
have

Let $M(t, \delta, \mu)=\mathbf{E} \max _{0 \leq s \leq t}\left|\boldsymbol{q}_{s}^{\mu, \delta}-\tilde{\boldsymbol{q}}_{s}^{\delta}\right|_{\mathbb{R}^{d}}$. By (3.4) and (3.6), using estimate (3.3), we

$$
M(t, \delta, \mu) \leq K_{1} \int_{0}^{t} M(s, \delta, \mu) d s+K_{2}(t, \delta) \int_{0}^{t} M(s, \delta, \mu) d s+o_{\mu}(1) .
$$

Here $o_{\mu}(1)$ is a term which goes to 0 as $\mu \downarrow 0$. The positive constant $K_{1}$ is independent of $\mu, \delta$ and $t$. The positive constant $K_{2}=K_{2}(t, \delta)$ may depend on $t$ and $\delta$, but is independent of $\mu$. Now we use the Bellman-Gronwall inequality:

$$
M(t, \delta, \mu) \leq o_{\mu}(1) \exp \left(\left(K_{1}+K_{2}(t, \delta)\right) t\right) .
$$

We conclude that for any $\delta, \kappa, T>0$ fixed and any $\boldsymbol{p}_{0}^{\mu, \delta}=\boldsymbol{p}$ fixed,

$$
\lim _{\mu \downarrow 0} \mathbf{P}\left(\max _{0 \leq t \leq T}\left|\boldsymbol{q}_{t}^{\mu, \delta}-\widetilde{\boldsymbol{q}}_{t}^{\delta}\right|_{\mathbb{R}^{d}}>\kappa\right)=0 .
$$

Now we can take $\delta \downarrow 0$. Using Theorem 6.7.2 from [10] we get the following result.

Theorem 3.1. We have, as $\delta \downarrow 0$, that

$$
\lim _{\delta \rightarrow 0} \mathbf{E} \max _{t \in[0, T]}\left|\widetilde{\boldsymbol{q}}_{t}^{\delta}-\widehat{\boldsymbol{q}}_{t}\right|_{\mathbb{R}^{d}}=0,
$$

where $\widehat{\boldsymbol{q}}_{t}$ is the solution of the problem

$$
\dot{\hat{\boldsymbol{q}}}_{t}=\frac{\boldsymbol{b}\left(\widehat{\boldsymbol{q}}_{t}\right)}{\lambda\left(\widehat{\boldsymbol{q}}_{t}\right)}+\frac{1}{\lambda\left(\widehat{\boldsymbol{q}}_{t}\right)} \circ \dot{\boldsymbol{W}}_{t}, \widehat{\boldsymbol{q}}_{0}=\boldsymbol{q} \in \mathbb{R}^{d} .
$$

Here the stochastic term is understood in the Stratonovich sense.

In the general case

$$
\mu \ddot{\boldsymbol{q}}_{t}^{\mu, \delta}=\boldsymbol{b}\left(\boldsymbol{q}_{t}^{\mu, \delta}\right)-\lambda\left(\boldsymbol{q}_{t}^{\mu, \delta}\right) \dot{\boldsymbol{q}}_{t}^{\mu, \delta}+\sigma\left(\boldsymbol{q}_{t}^{\mu, \delta}\right) \dot{\boldsymbol{W}}_{t}^{\delta}, \boldsymbol{q}_{0}^{\mu, \delta}=\boldsymbol{q}, \dot{\boldsymbol{q}}_{0}^{\mu, \delta}=\boldsymbol{p},
$$

where the matrix $\sigma(\bullet)$ satisfy assumptions made in Section 1, we have, similarly, that for any $\delta, \kappa, T>0$ fixed and any $\boldsymbol{p}_{0}^{\mu, \delta}=\boldsymbol{p}$ fixed,

$$
\lim _{\mu \downarrow 0} \mathbf{P}\left(\max _{0 \leq t \leq T}\left|\boldsymbol{q}_{t}^{\mu, \delta}-\widetilde{\boldsymbol{q}}_{t}^{\delta}\right|_{\mathbb{R}^{d}}>\kappa\right)=0 .
$$

The process $\widetilde{\boldsymbol{q}}_{t}^{\delta}$ is governed by the equation 


$$
\dot{\boldsymbol{q}}_{t}^{\delta}=\frac{\boldsymbol{b}\left(\widetilde{\boldsymbol{q}}_{t}^{\delta}\right)}{\lambda\left(\widetilde{\boldsymbol{q}}_{t}^{\delta}\right)}+\frac{\sigma\left(\widetilde{\boldsymbol{q}}_{t}^{\delta}\right)}{\lambda\left(\widetilde{\boldsymbol{q}}_{t}^{\delta}\right)} \dot{\boldsymbol{W}}_{t}^{\delta}, \widetilde{\boldsymbol{q}}_{0}^{\delta}=\boldsymbol{q} \in \mathbb{R}^{d}
$$

And we conclude with

Theorem 3.2. Under the assumptions mentioned above,

$$
\lim _{\delta \rightarrow 0} \mathbf{E} \max _{t \in[0, T]}\left|\widetilde{\boldsymbol{q}}_{t}^{\delta}-\widehat{\boldsymbol{q}}_{t}\right|_{\mathbb{R}^{d}}=0,
$$

where $\widehat{\boldsymbol{q}}_{t}$ is the solution of the problem

$$
\dot{\hat{\boldsymbol{q}}}_{t}=\frac{\boldsymbol{b}\left(\widehat{\boldsymbol{q}}_{t}\right)}{\lambda\left(\widehat{\boldsymbol{q}}_{t}\right)}+\frac{\sigma\left(\widehat{\boldsymbol{q}}_{t}\right)}{\lambda\left(\widehat{\boldsymbol{q}}_{t}\right)} \circ \dot{\boldsymbol{W}}_{t}, \widehat{\boldsymbol{q}}_{0}=\boldsymbol{q} \in \mathbb{R}^{d} .
$$

\section{One dimensional case}

In the case of one space variable, Smoluchowski-Kramers approximation leads to an one-dimensional diffusion process $q_{t}$ which is defined by the following stochastic differential equation written in the Itô form:

$$
\dot{q}_{t}=\frac{b\left(q_{t}\right)}{\lambda\left(q_{t}\right)}-\frac{\lambda^{\prime}\left(q_{t}\right)}{2 \lambda^{3}\left(q_{t}\right)}+\frac{1}{\lambda\left(q_{t}\right)} \dot{W}_{t}, q_{0}=q \in \mathbb{R}^{1}
$$

Put

$$
\begin{aligned}
& u(q)=\int_{0}^{q} \lambda(x) \exp \left(-2 \int_{0}^{x} b(y) \lambda(y) d y\right) d x, \\
& v(q)=2 \int_{0}^{q} \lambda(x) \exp \left(2 \int_{0}^{x} b(y) \lambda(y) d y\right) d x .
\end{aligned}
$$

Since $\lambda(x)>0, u(q)$ and $v(q)$ are strictly increasing functions. Following [4] we introduce an operator $D_{v} D_{u}$, where $D_{u}$ means the differentiation with respect to the monotone function $u(q): D_{u} f(q)=\lim _{h \rightarrow 0} \frac{f(x+h)-f(x)}{u(x+h)-u(x)}$; the operator $D_{v}$ is defined in a similar way. One can check that $D_{v} D_{u}$ is the generator of the diffusion process $q_{t}$ defined by (4.1).

Suppose now that the friction coefficient $\lambda(q)=\lambda_{\varepsilon}(q)$ depends on a parameter $\varepsilon>0$. We assume that, for each $\varepsilon \in(0,1], \lambda_{\varepsilon}(q)$ has a bounded continuous derivative $\lambda_{\varepsilon}^{\prime}(q)$, and $0<\underline{\lambda} \leq \lambda_{\varepsilon}(q) \leq \bar{\lambda}<\infty$. Let $u_{\varepsilon}(q)$ and $v_{\varepsilon}(q)$ be the functions defined by (4.2) when $\lambda(q)$ is replaced by $\lambda_{\varepsilon}(q)$.

Consider the stochastic process $q_{t}^{\mu, \delta, \varepsilon}$ in $\mathbb{R}^{1}$ defined by the equation

$$
\mu \ddot{q}_{t}^{\mu, \delta, \varepsilon}=b\left(q_{t}^{\mu, \delta, \varepsilon}\right)-\lambda^{\varepsilon}\left(q_{t}^{\mu, \delta, \varepsilon}\right) q_{t}^{\mu, \delta, \varepsilon}+\dot{W}_{t}^{\delta}, q_{0}^{\mu, \delta, \varepsilon}=q, \dot{q}_{0}^{\mu, \delta, \varepsilon}=p
$$


where $\dot{W}_{t}^{\delta}$ is, as before, a "smoothed" white noise converging to $\dot{W}_{t}$ as $\delta \downarrow 0$.

Theorem 4.1. Assume that the function $\lambda_{\varepsilon}(q)$ converge weakly as $\varepsilon \downarrow 0$ on each finite interval $[\alpha, \beta] \subset \mathbb{R}^{1}$ to a function $\bar{\lambda}(q)$ (maybe, discontinuous). Then processes $q_{t}^{\mu, \delta, \varepsilon}$ converge weakly on each finite time interval to the diffusion process $\bar{q}_{t}$ governed by the generator $D_{\bar{v}} D_{\bar{u}}$ (where $\bar{u}(q)$ and $\bar{v}(q)$ defined by (4.2) with $\lambda=\bar{\lambda}(q)$ ) as, first $\mu \downarrow 0$, then $\delta \downarrow 0$, and then $\varepsilon \downarrow 0$.

Proof. According to Section 3, processes $q_{t}^{\mu, \delta, \varepsilon}$ converge weakly as first $\mu \downarrow 0$ and then $\delta \downarrow 0$ to the process $\widehat{q}_{t}^{\delta}$ which solves equation (4.1) with $\lambda(q)=\lambda^{\varepsilon}(q)$. It follows from our assumptions that functions $u_{\varepsilon}(q)$ and $v_{\varepsilon}(q)$ converge as $\varepsilon \downarrow 0$ to functions $\bar{u}(q)$ and $\bar{v}(q)$ respectively for each $q \in \mathbb{R}^{1}$. The functions $\bar{u}(q)$ and $\bar{v}(q)$ are continuous and strictly increasing. Therefore ([4]) a diffusion process $\bar{q}_{t}$ exists governed by $D_{\bar{v}} D_{\bar{u}}$. As shown in [8], convergence of $u_{\varepsilon}(q)$ and $v_{\varepsilon}(q)$ as $\varepsilon \downarrow 0$ to $\bar{u}(q)$ and $\bar{v}(q)$ respectively implies weak convergence of processes $q_{t}^{\varepsilon}$ to the process corresponding to $D_{\bar{v}} D_{\bar{u}}$ as $\varepsilon \downarrow 0$.

Theorem 4.2. Let $\lambda_{\varepsilon}(q)=\tilde{\lambda}\left(\frac{q}{\varepsilon}\right)$. Assume that one of the following conditions is satisfied:

1. $\widetilde{\lambda}(q)$ is a continuously differentiable positive 1-periodic function;

2. $\widetilde{\lambda}(q)$ is an ergodic stationary process (independent of the process $W_{t}$ in (4.3)) with continuously differentiable trajectories and $0<\lambda_{-} \leq \widetilde{\lambda}(q) \leq \lambda_{+}<\infty$ for some constants $\lambda_{-}, \lambda_{+}$. satisfied.

Put $\bar{\lambda}=\int_{0}^{1} \widetilde{\lambda}(q) d q$ if condition 1 is satisfied, and $\bar{\lambda}=\mathbf{E} \widetilde{\lambda}(q)$ is condition 2 is

Then the process $q_{t}^{\mu, \delta, \varepsilon}$ defined by (5.3) converge weakly when first $\mu \downarrow 0$ and then $\varepsilon \downarrow 0$ to the process $\bar{q}_{t}$ defined by the equation

$$
\bar{q}_{t}=\frac{1}{\bar{\lambda}} b\left(\bar{q}_{t}\right)+\frac{1}{\bar{\lambda}} \dot{W}_{t}, \bar{q}_{0}=q .
$$

Proof of this theorem follows from Theorem 4.1 since each of conditions 1 and 2 implies conditions of Theorem 4.1 and $\bar{\lambda}(q)=\bar{\lambda}$.

Assume now that $\lambda_{\varepsilon}(q)$ is a bounded and separated from zero uniformly in $\varepsilon \in(0,1]$ positive function such that $\lim _{\varepsilon \downarrow 0} \lambda_{\varepsilon}(q)=\lambda_{1}$ for $q<0$, and $\lim _{\varepsilon \downarrow 0} \lambda_{\varepsilon}(q)=\lambda_{2}$ for $q>0$. Assume that $\lambda_{\varepsilon}(q)$ is continuously differentiable for each $\varepsilon>0$. Let $\widehat{\lambda}(q)$ be the step function equal to $\lambda_{1}$ for $q \leq 0$ and to $\lambda_{2}$ for $q>0$. Let functions $\widehat{u}(q)$ and $\widehat{v}(q)$ be defined by formula (4.2) with $\lambda(q)=\widehat{\lambda}(q)$; $\widehat{u}(q)$ and $\widehat{v}(q)$ are continuous strictly 
increasing functions. Denote by $\widehat{q}_{t}$ the diffusion process in $\mathbb{R}^{1}$ governed by the generator $A=D_{\widehat{v}} D_{\widehat{u}}$. The process $\widehat{q}_{t}$ behaves as $\frac{1}{\lambda_{1}} W_{t}$ on the negative part of axis $q$ and as $\frac{1}{\lambda_{2}} W_{t}$ on the positive part. Its behavior at $q=0$ is defined by the domain of definition $\mathfrak{D}_{A}$ of the generator $A$ : a continuous bounded function $f(q), q \in \mathbb{R}^{1}$, twice continuously differentiable at $q \in\left\{\mathbb{R}^{1} \backslash\{q=0\}\right\}$ belongs to $\mathfrak{D}_{A}$ if and only if left and right derivatives at $q=0, f_{-}^{\prime}(0)$ and $f_{+}^{\prime}(0)$ respectively, satisfy the equality $\frac{1}{\lambda_{1}} f_{-}^{\prime}(0)=\frac{1}{\lambda_{2}} f_{+}^{\prime}(0)$ and $A f(q)$ is continuous.

It is easy to see that functions $u_{\varepsilon}(q)$ and $v_{\varepsilon}(q)$ defined by (4.2) with $\lambda(q)=\lambda_{\varepsilon}(q)$ converge as $\varepsilon \downarrow 0$ to $\widehat{u}(q)$ and $\widehat{v}(q)$ respectively for each $q \in \mathbb{R}^{1}$. This implies the following result.

Theorem 4.3. Let the friction coefficient $\lambda_{\varepsilon}(q)$ satisfies the conditions mentioned above. Then the stochastic process $q_{t}^{\mu, \delta, \varepsilon}$ defined by (4.3) converges weakly to the diffusion process $\widehat{q}_{t}$ in $\mathbb{R}^{1}$ governed by $A=D_{\widehat{v}} D_{\widehat{u}}$ as first $\mu \downarrow 0$, then $\delta \downarrow 0$, and then $\varepsilon \downarrow 0$.

This means, roughly speaking, that, if the friction coefficient is close to the stepfunction $\widehat{\lambda}(q)$, then process $q_{t}^{\mu}$, for $0<\mu<<1$, can be approximated by the diffusion process $\widehat{q}_{t}$.

\section{Multidimensional case}

In this section we consider the problem of fast oscillating periodic environment in multidimensional case. We consider the system

$$
\mu \ddot{\boldsymbol{q}}_{t}^{\mu, \delta, \varepsilon}=\boldsymbol{b}\left(\frac{\boldsymbol{q}_{t}^{\mu, \delta, \varepsilon}}{\varepsilon}\right)-\lambda\left(\frac{\boldsymbol{q}_{t}^{\mu, \delta, \varepsilon}}{\varepsilon}\right) \dot{\boldsymbol{q}}_{t}^{\mu, \delta, \varepsilon}+\dot{\boldsymbol{W}}_{t}^{\delta}, \boldsymbol{q}_{0}^{\mu, \delta, \varepsilon}=\boldsymbol{q} \in \mathbb{R}^{d}, \dot{\boldsymbol{q}}_{0}^{\mu, \delta, \varepsilon}=\boldsymbol{p} \in \mathbb{R}^{d}
$$

Here as in Section 3 the process $\boldsymbol{W}_{t}^{\delta}$ is the approximation of the Wiener process in $\mathbb{R}^{d}$. We make the same assumptions about the functions $\lambda(\bullet)$ and $\boldsymbol{b}(\bullet)$ as in Section 2. In addition we assume that the functions $\lambda(\bullet)$ and $\boldsymbol{b}(\bullet)$ are 1-periodic, i.e. $\lambda\left(\boldsymbol{x}+\mathbf{e}_{k}\right)=\lambda(\boldsymbol{x})$ and $\boldsymbol{b}\left(\boldsymbol{x}+\mathbf{e}_{k}\right)=\boldsymbol{b}(\boldsymbol{x})$ for $\boldsymbol{x} \in \mathbb{R}^{d}$ and $\mathbf{e}_{k}=(0,0, \ldots, 1(k$-th coordinate $), \ldots, 0), 1 \leq k \leq d$. Under this assumption our system (5.1) could be regarded as a system on the $d$-torus $\mathbb{T}^{d}=\mathbb{R}^{d} / \mathbb{Z}^{d}$. Fix $\varepsilon>0$, we can proceed as in Section 3 to see that first as $\mu \downarrow 0$ then as $\delta \downarrow 0$ the process $\boldsymbol{q}_{t}^{\mu, \delta, \varepsilon}$ converges in probability to the process $\boldsymbol{q}_{t}^{\varepsilon}$ subjected to

$$
\dot{\boldsymbol{q}}_{t}^{\varepsilon}=\frac{\boldsymbol{b}\left(\frac{\boldsymbol{q}_{t}^{\varepsilon}}{\varepsilon}\right)}{\lambda\left(\frac{\boldsymbol{q}_{t}^{\varepsilon}}{\varepsilon}\right)}+\frac{1}{\lambda\left(\frac{\boldsymbol{q}_{t}^{\varepsilon}}{\varepsilon}\right)} \circ \dot{\boldsymbol{W}}_{t}, \boldsymbol{q}_{0}^{\varepsilon}=\boldsymbol{q} \in \mathbb{R}^{d} .
$$


The above equation, written in the form of Itô integral, will be

$$
\dot{\boldsymbol{q}}_{t}^{\varepsilon}=\frac{\boldsymbol{b}\left(\frac{\boldsymbol{q}_{t}^{\varepsilon}}{\varepsilon}\right)}{\lambda\left(\frac{\boldsymbol{q}_{t}^{\varepsilon}}{\varepsilon}\right)}-\frac{1}{2 \varepsilon} \frac{\nabla \lambda\left(\frac{\boldsymbol{q}_{t}^{\varepsilon}}{\varepsilon}\right)}{\lambda^{3}\left(\frac{\boldsymbol{q}_{t}^{\varepsilon}}{\varepsilon}\right)}+\frac{1}{\lambda\left(\frac{\boldsymbol{q}_{t}^{\varepsilon}}{\varepsilon}\right)} \dot{\boldsymbol{W}}_{t}, \boldsymbol{q}_{0}^{\varepsilon}=\boldsymbol{q} \in \mathbb{R}^{d} .
$$

The generator corresponding to (5.2) is the second order differential operator

$$
L^{\varepsilon} u(\boldsymbol{x})=\left(\frac{\boldsymbol{b}\left(\frac{\boldsymbol{x}}{\varepsilon}\right)}{\lambda\left(\frac{\boldsymbol{x}}{\varepsilon}\right)}-\frac{1}{2 \varepsilon} \frac{\nabla \lambda\left(\frac{\boldsymbol{x}}{\varepsilon}\right)}{\lambda^{3}\left(\frac{\boldsymbol{x}}{\varepsilon}\right)}\right) \cdot \nabla u(\boldsymbol{x})+\frac{1}{2} \frac{1}{\lambda^{2}\left(\frac{\boldsymbol{x}}{\varepsilon}\right)} \Delta u(\boldsymbol{x}) .
$$

Our goal is to study the homogenization properties of (5.3) for general multidimensional case. Homogenization problems are considered by many authors, see, e.g., [7], [15], [14], [13], [12]. However, we provide here an elementary probabilistic way of doing this. Our method follows [7] and [6](pp. 104-106).

Let us first make a change of variable $\frac{\boldsymbol{q}}{\varepsilon}=\boldsymbol{y}$ and $\frac{t}{\varepsilon^{2}}=s$. The process $\boldsymbol{y}_{s}^{\varepsilon}=\frac{1}{\varepsilon} \boldsymbol{q}_{t}^{\varepsilon}$ corresponds to the generator

$$
A^{\varepsilon}=\frac{1}{2 \lambda^{2}(\boldsymbol{y})} \Delta_{\boldsymbol{y}}-\frac{\nabla \lambda(\boldsymbol{y})}{2 \lambda^{3}(\boldsymbol{y})} \cdot \nabla_{\boldsymbol{y}}+\varepsilon \frac{\boldsymbol{b}(\boldsymbol{y})}{\lambda(\boldsymbol{y})} \cdot \nabla_{\boldsymbol{y}}
$$

We regard $\boldsymbol{y}_{s}^{\varepsilon}$ as a process on $\mathbb{T}^{d}$. Then we have the bound

$$
\left|\mathbf{E}_{\boldsymbol{q} / \varepsilon} f\left(\boldsymbol{y}_{s}^{\varepsilon}\right)-\int_{\mathbb{T}^{d}} f(\boldsymbol{x}) \mu^{\varepsilon}(\boldsymbol{x}) d \boldsymbol{x}\right|<K e^{-a s} .
$$

Here $K>0$ and $a>0$ are independent of $\varepsilon$ for small $\varepsilon$. The function $f$ is bounded and measurable. The function $\mu^{\varepsilon}(\boldsymbol{x})$ is the density of the unique invariant measure of $\boldsymbol{y}_{s}^{\varepsilon}$ on $\mathbb{T}^{d}$ and $\int_{\mathbb{T}^{d}} \mu^{\varepsilon}(\boldsymbol{x}) d \boldsymbol{x}=1$. We have

$$
\lim _{\varepsilon \downarrow 0} \mu^{\varepsilon}(\boldsymbol{x})=\mu(\boldsymbol{x}), \lim _{\varepsilon \downarrow 0} \int_{\mathbb{T}^{d}} f(\boldsymbol{x}) \mu^{\varepsilon}(\boldsymbol{x}) d \boldsymbol{x}=\int_{\mathbb{T}^{d}} f(\boldsymbol{x}) \mu(\boldsymbol{x}) d \boldsymbol{x}
$$

for $f \in C\left(\mathbb{T}^{d}\right)$ and $\mu(\boldsymbol{x})$ the unique invariant measure for the process with generator $A^{0}$ on $\mathbb{T}^{d}$ and $\int_{\mathbb{T}^{d}} \mu(\boldsymbol{x}) d \boldsymbol{x}=1$. Combining these estimates we have, that for any $n$, for any $t \geq \delta>0$, there exist $\varepsilon_{0}(n, \delta)>0$ such that for any $0<\varepsilon<\varepsilon_{0}(n, \delta)$, we have

$$
\left|\mathbf{E}_{\boldsymbol{q}} f\left(\frac{\boldsymbol{q}_{t}^{\varepsilon}}{\varepsilon}\right)-\int_{\mathbb{T}^{d}} f(\boldsymbol{x}) \mu(\boldsymbol{x}) d \boldsymbol{x}\right|<\frac{1}{n} .
$$

This implies that for any $f \in C\left(\mathbb{T}^{d}\right)$,

$$
\limsup _{\varepsilon \downarrow 0}\left|\mathbf{E}_{\boldsymbol{q}} f\left(\frac{\boldsymbol{q}_{t}^{\varepsilon}}{\varepsilon}\right)-\int_{\mathbb{T}^{d}} f(\boldsymbol{x}) \mu(\boldsymbol{x}) d \boldsymbol{x}\right|=0 .
$$


Finally we calculate the density $\mu(\boldsymbol{x})$. Since

$$
A^{0}=\frac{1}{2 \lambda^{2}(\boldsymbol{y})} \Delta_{\boldsymbol{y}}-\frac{\nabla \lambda(\boldsymbol{y})}{2 \lambda^{3}(\boldsymbol{y})} \cdot \nabla_{\boldsymbol{y}}=\frac{1}{2 \lambda^{2}(\boldsymbol{y})}\left(\Delta_{\boldsymbol{y}}-\nabla(\ln \lambda(\boldsymbol{y})) \cdot \nabla_{\boldsymbol{y}}\right)
$$

we see that $\mu(\boldsymbol{x})=C \lambda(\boldsymbol{x})$ with $C=\left(\int_{\mathbb{T}^{d}} \lambda(\boldsymbol{x}) d \boldsymbol{x}\right)^{-1}$ and we have the following result:

Lemma 5.1. For any $f \in C\left(\mathbb{T}^{d}\right)$, we have

$$
\limsup _{\varepsilon \downarrow 0}\left|\mathbf{E}_{t \geq \delta} f\left(\frac{\boldsymbol{q}_{t}^{\varepsilon}}{\varepsilon}\right)-\frac{\int_{\mathbb{T}^{d}} f(\boldsymbol{x}) \lambda(\boldsymbol{x}) d \boldsymbol{x}}{\int_{\mathbb{T}^{d}} \lambda(\boldsymbol{x}) d \boldsymbol{x}}\right|=0 .
$$

Corollary. For any bounded continuous function $f(\boldsymbol{x})$ on $\mathbb{T}^{d}, \boldsymbol{q} \in \mathbb{T}^{d}$ we have

$$
\mathbf{E}_{\boldsymbol{q}}\left[\int_{0}^{t} f\left(\frac{\boldsymbol{q}_{s}^{\varepsilon}}{\varepsilon}\right) d s-\frac{t \int_{\mathbb{T}^{d}} f(\boldsymbol{x}) \lambda(\boldsymbol{x}) d \boldsymbol{x}}{\int_{\mathbb{T}^{d}} \lambda(\boldsymbol{x}) d \boldsymbol{x}}\right]^{2} \rightarrow 0
$$

as $\varepsilon \downarrow 0$, for $0<t<\infty$.

The proof of this corollary follows the same proof of the corollary after Lemma 1 in $[7]$.

Now let us consider auxiliary functions $N_{k}(\boldsymbol{y}), k=1, \ldots, d$, which are the periodic bounded solutions (i.e., on $\mathbb{T}^{d}$ ) of the equations

$$
\frac{1}{2 \lambda^{2}(\boldsymbol{y})} \Delta_{\boldsymbol{y}} N_{k}(\boldsymbol{y})-\frac{\nabla_{\boldsymbol{y}} \lambda(\boldsymbol{y})}{2 \lambda^{3}(\boldsymbol{y})} \cdot \nabla_{\boldsymbol{y}} N_{k}(\boldsymbol{y})=A^{0}\left(N_{k}(\boldsymbol{y})\right)=\frac{1}{2 \lambda^{3}(\boldsymbol{y})} \frac{\partial \lambda}{\partial y_{k}}(\boldsymbol{y}), \boldsymbol{y} \in \mathbb{T}^{d}
$$

The solvability of this equation comes from the fact that $\left(A^{0}\right)^{*} \lambda(\boldsymbol{y})=0$ and $\int_{\mathbb{T}^{d}} \frac{1}{2 \lambda^{3}(\boldsymbol{y})} \frac{\partial \lambda}{\partial y_{k}}(\boldsymbol{y}) \lambda(\boldsymbol{y}) d \boldsymbol{y}=0$. The boundedness of solution comes from our assumptions on the function $\lambda(\bullet)$. Now we apply Itô's formula: 


$$
\begin{aligned}
& \varepsilon N_{k}\left(\frac{\boldsymbol{q}_{t}^{\varepsilon}}{\varepsilon}\right)-\varepsilon N_{k}\left(\frac{\boldsymbol{q}}{\varepsilon}\right) \\
& =\varepsilon\left[\int_{0}^{t} \nabla N_{k}\left(\frac{\boldsymbol{q}_{s}^{\varepsilon}}{\varepsilon}\right) \cdot \frac{1}{\varepsilon}\left(\frac{\boldsymbol{b}}{\lambda}\left(\frac{\boldsymbol{q}_{s}^{\varepsilon}}{\varepsilon}\right)-\frac{1}{2 \varepsilon} \frac{\nabla \lambda}{\lambda^{3}}\left(\frac{\boldsymbol{q}_{s}^{\varepsilon}}{\varepsilon}\right)+\frac{\dot{\boldsymbol{W}}_{s}}{\lambda\left(\frac{\boldsymbol{q}_{s}^{\varepsilon}}{\varepsilon}\right)}\right) d s+\right. \\
& \left.+\frac{1}{2} \int_{0}^{t} \Delta N_{k}\left(\frac{\boldsymbol{q}_{s}^{\varepsilon}}{\varepsilon}\right) \frac{1}{\varepsilon^{2}} \frac{1}{\lambda^{2}\left(\frac{\boldsymbol{q}_{s}^{\varepsilon}}{\varepsilon}\right)} d s\right] \\
& =\int_{0}^{t} \nabla N_{k}\left(\frac{\boldsymbol{q}_{s}^{\varepsilon}}{\varepsilon}\right) \cdot\left(\frac{\boldsymbol{b}}{\lambda}\left(\frac{\boldsymbol{q}_{s}^{\varepsilon}}{\varepsilon}\right)+\frac{\dot{\boldsymbol{W}}_{s}}{\lambda\left(\frac{\boldsymbol{q}_{s}^{\varepsilon}}{\varepsilon}\right)}\right) d s+\frac{1}{2 \varepsilon} \int_{0}^{t} \frac{\frac{\partial \lambda}{\partial y_{k}}\left(\frac{\boldsymbol{q}_{s}^{\varepsilon}}{\varepsilon}\right)}{\lambda^{3}\left(\frac{\boldsymbol{q}_{s}^{\varepsilon}}{\varepsilon}\right)} d s .
\end{aligned}
$$

Let $\boldsymbol{N}(\boldsymbol{y})=\left(N_{1}(\boldsymbol{y}), \ldots, N_{d}(\boldsymbol{y})\right)$. Using (5.5) we have

$$
\begin{aligned}
\boldsymbol{q}_{t}^{\varepsilon}-\boldsymbol{q}=\int_{0}^{t} & \left(\frac{\boldsymbol{b}}{\lambda}\left(\frac{\boldsymbol{q}_{s}^{\varepsilon}}{\varepsilon}\right)+\frac{\dot{\boldsymbol{W}}_{s}}{\lambda\left(\frac{\boldsymbol{q}_{s}^{\varepsilon}}{\varepsilon}\right)}\right) d s+\int_{0}^{t}(D \boldsymbol{N})\left(\frac{\boldsymbol{q}_{s}^{\varepsilon}}{\varepsilon}\right)\left(\frac{\boldsymbol{b}}{\lambda}\left(\frac{\boldsymbol{q}_{s}^{\varepsilon}}{\varepsilon}\right)+\frac{\dot{\boldsymbol{W}}_{s}}{\lambda\left(\frac{\boldsymbol{q}_{s}^{\varepsilon}}{\varepsilon}\right)}\right) d s+ \\
& -\varepsilon\left(\boldsymbol{N}\left(\frac{\boldsymbol{q}_{t}^{\varepsilon}}{\varepsilon}\right)-\boldsymbol{N}\left(\frac{\boldsymbol{q}}{\varepsilon}\right)\right) ;
\end{aligned}
$$

Here $(D \boldsymbol{N})(\boldsymbol{y})=\left(\frac{\partial N_{i}}{\partial y_{j}}\right)_{1 \leq i, j \leq d}, \boldsymbol{y}=\left(y_{1}, \ldots, y_{d}\right) \in \mathbb{T}^{d}$.

Therefore using the corollary after Lemma 1 , we see that $\boldsymbol{q}_{t}^{\varepsilon}$ converges weakly to a process $\boldsymbol{q}_{t}, \boldsymbol{q}_{0}=\boldsymbol{q} \in \mathbb{R}^{d}$ governed by the operator

$$
\bar{L}=\frac{1}{2} \sum_{i, j=1}^{d} \bar{a}_{i j} \frac{\partial^{2}}{\partial y_{i} \partial y_{j}}+\sum_{i=1}^{d} \bar{b}_{i} \frac{\partial}{\partial y_{i}},
$$

with coefficients

$$
\begin{gathered}
\bar{a}_{i j}=\int_{\mathbb{T}^{d}}\left(\frac{\nabla N_{i}(\boldsymbol{y}) \cdot \nabla N_{j}(\boldsymbol{y})}{\lambda(\boldsymbol{y})}+\frac{1}{\lambda(\boldsymbol{y})}\left(\frac{\partial N_{j}}{\partial y_{i}}(\boldsymbol{y})+\frac{\partial N_{i}}{\partial y_{j}}(\boldsymbol{y})\right)+\delta_{i j} \frac{1}{\lambda(\boldsymbol{y})}\right) d \boldsymbol{y} /\left(\int_{\mathbb{T}^{d}} \lambda(\boldsymbol{y}) d \boldsymbol{y}\right), \\
\bar{b}_{i}=\frac{\int_{\mathbb{T}^{d}} b_{i}(\boldsymbol{y}) d \boldsymbol{y}}{\int_{\mathbb{T}^{d}} \lambda(\boldsymbol{y}) d \boldsymbol{y}}+\sum_{k=1}^{d} \frac{\int_{\mathbb{T}^{d}} b_{k}(\boldsymbol{y}) \frac{\partial N_{i}}{\partial y_{k}}(\boldsymbol{y}) d \boldsymbol{y}}{\int_{\mathbb{T}^{d}} \lambda(\boldsymbol{y}) d \boldsymbol{y}}
\end{gathered}
$$

Here $\delta_{i j}=1$ if $i=j$, and $\delta_{i j}=0$ otherwise.

We could simplify the expression for $\bar{a}_{i j}$ : using (5.5) we get 


$$
\begin{aligned}
& \bar{a}_{i j}=\int_{\mathbb{T}^{d}}\left(\frac{\nabla N_{i}(\boldsymbol{y}) \cdot \nabla N_{j}(\boldsymbol{y})}{\lambda(\boldsymbol{y})}+\frac{1}{\lambda(\boldsymbol{y})}\left(\frac{\partial N_{j}}{\partial y_{i}}(\boldsymbol{y})+\frac{\partial N_{i}}{\partial y_{j}}(\boldsymbol{y})\right)+\delta_{i j} \frac{1}{\lambda(\boldsymbol{y})}\right) d \boldsymbol{y} /\left(\int_{\mathbb{T}^{d}} \lambda(\boldsymbol{y}) d \boldsymbol{y}\right) \\
& =\int_{\mathbb{T}^{d}}\left(\operatorname{div}\left(\frac{N_{i}(\boldsymbol{y})}{\lambda(\boldsymbol{y})} \nabla N_{j}(\boldsymbol{y})\right)-\frac{N_{i}(\boldsymbol{y})}{\lambda(\boldsymbol{y})} \Delta N_{j}(\boldsymbol{y})-N_{i}(\boldsymbol{y}) \nabla N_{j}(\boldsymbol{y}) \cdot \nabla\left(\frac{1}{\lambda(\boldsymbol{y})}\right)+\right. \\
& \left.+\frac{1}{\lambda(\boldsymbol{y})}\left(\frac{\partial N_{j}}{\partial y_{i}}(\boldsymbol{y})+\frac{\partial N_{i}}{\partial y_{j}}(\boldsymbol{y})\right)+\delta_{i j} \frac{1}{\lambda(\boldsymbol{y})}\right) d \boldsymbol{y} /\left(\int_{\mathbb{T}^{d}} \lambda(\boldsymbol{y}) d \boldsymbol{y}\right) \\
& =\int_{\mathbb{T}^{d}}\left(\frac{\partial}{\partial y_{j}}\left(N_{i}(\boldsymbol{y}) \frac{1}{\lambda(\boldsymbol{y})}\right)-\frac{1}{\lambda(\boldsymbol{y})} \frac{\partial N_{i}}{\partial y_{j}}(\boldsymbol{y})+\right. \\
& \left.+\frac{1}{\lambda(\boldsymbol{y})}\left(\frac{\partial N_{j}}{\partial y_{i}}(\boldsymbol{y})+\frac{\partial N_{i}}{\partial y_{j}}(\boldsymbol{y})\right)+\delta_{i j} \frac{1}{\lambda(\boldsymbol{y})}\right) d \boldsymbol{y} /\left(\int_{\mathbb{T}^{d}} \lambda(\boldsymbol{y}) d \boldsymbol{y}\right) \\
& =\frac{\int_{\mathbb{T}^{d}} \frac{\partial N_{j}}{\partial y_{i}}(\boldsymbol{y}) \frac{1}{\lambda(\boldsymbol{y})} d \boldsymbol{y}}{\int_{\mathbb{T}^{d}} \lambda(\boldsymbol{y}) d \boldsymbol{y}}+\delta_{i j} \frac{\int_{\mathbb{T}^{d}} \frac{1}{\lambda(\boldsymbol{y})} d \boldsymbol{y}}{\int_{\mathbb{T}^{d}} \lambda(\boldsymbol{y}) d \boldsymbol{y}} .
\end{aligned}
$$

So we have

Theorem 5.1. As $\varepsilon \downarrow 0$, the process $\boldsymbol{q}_{t}^{\varepsilon}$ converges weakly to a process $\boldsymbol{q}_{t}, \boldsymbol{q}_{0}=\boldsymbol{q} \in$ $\mathbb{R}^{d}$ governed by the operator (5.7) with coefficients given by (5.8) and (5.9).

This Theorem implies a homogenization result for the process $\boldsymbol{q}_{t}^{\mu, \delta, \varepsilon}$ defined by equation (5.1).

\section{Remarks and Generalizations}

\subsection{Small mass - large friction asymptotics.}

Let the friction coefficient in (1.1) be $\lambda^{\varepsilon}(\boldsymbol{q})=\varepsilon^{-1} \lambda(\boldsymbol{q}), \boldsymbol{q} \in \mathbb{R}^{n}, 0<\varepsilon<<1$. As it follows from Theorem 3.1, the Smoluchowski-Kramers approximation in this case has the form:

$$
\dot{\overline{\boldsymbol{q}}}_{t}^{\varepsilon}=\frac{\varepsilon \boldsymbol{b}\left(\overline{\boldsymbol{q}}_{t}^{\varepsilon}\right)}{\lambda\left(\overline{\boldsymbol{q}}_{t}^{\varepsilon}\right)}-\frac{\varepsilon^{2} \nabla \lambda\left(\overline{\boldsymbol{q}}_{t}^{\varepsilon}\right)}{2 \lambda^{3}\left(\overline{\boldsymbol{q}}_{t}^{\varepsilon}\right)}+\frac{\varepsilon}{\lambda\left(\overline{\boldsymbol{q}}_{t}^{\varepsilon}\right)} \dot{\boldsymbol{W}}_{t}, \overline{\boldsymbol{q}}_{0}^{\varepsilon}=\boldsymbol{q} .
$$

Put $\widetilde{\boldsymbol{q}}_{t}^{\varepsilon}=\overline{\boldsymbol{q}}_{t / \varepsilon}^{\varepsilon}$. Then $\widetilde{\boldsymbol{q}}_{t}^{\varepsilon}$ satisfies the equation

$$
\dot{\tilde{\boldsymbol{q}}}_{t}^{\varepsilon}=\frac{\boldsymbol{b}\left(\widetilde{\boldsymbol{q}}_{t}^{\varepsilon}\right)}{\lambda\left(\widetilde{\boldsymbol{q}}_{t}^{\varepsilon}\right)}-\frac{\varepsilon \nabla \lambda\left(\widetilde{\boldsymbol{q}}_{t}^{\varepsilon}\right)}{2 \lambda^{3}\left(\widetilde{\boldsymbol{q}}_{t}^{\varepsilon}\right)}+\frac{\sqrt{\varepsilon}}{\lambda\left(\widetilde{\boldsymbol{q}}_{t}^{\varepsilon}\right)} \dot{\overrightarrow{\boldsymbol{W}}}_{t}, \widetilde{\boldsymbol{q}}_{0}^{\varepsilon}=\boldsymbol{q},
$$

where $\widetilde{\boldsymbol{W}}_{t}$ is a Wiener process.

Assume that the vector field $\boldsymbol{b}(\boldsymbol{q}), \boldsymbol{q} \in \mathbb{R}^{n}$, has a finite number of compact attractors $K_{1}, \ldots, K_{l}$. Let, for brevity, each $K_{i}$ be an asymptotically stable equilibrium, and each point of $\mathbb{R}^{n}$, besides a separatrix set $\mathcal{E} \subset \mathbb{R}^{n}$, is attracted to one of these equilibriums. 
The separatrix set $\mathcal{E}$ is assumed to have dimension less than $n$. Then, if $\widetilde{\boldsymbol{q}}_{0}^{\varepsilon}=\boldsymbol{q} \notin \mathcal{E}, \widetilde{\boldsymbol{q}}_{t}^{\varepsilon}$ first comes to a small neighborhood of a stable equilibrium $K_{i}, i=i(\boldsymbol{q})$, with the probability close to 1 as $\varepsilon \downarrow 0$ and spends in this neighborhood a long time. Then, because of the large deviations, the trajectory will switch to the neighborhood of another attractor, then to another, and so on. We see from (6.1), that the long-time behavior of the system with a large friction is similar to the behavior of a system with a small noise. Applying the results of [9], Chapters 4,6 , we see that, for $0<\varepsilon<<1$, the sequence of transitions between the attractors, the main term of transition time logarithmic asymptotics, and the most probable transition paths are not random for generic systems. These characteristics of the long-time behavior are defined by a function $V(\boldsymbol{x}, \boldsymbol{y})$ :

$$
V(\boldsymbol{x}, \boldsymbol{y})=\inf \left\{\frac{1}{2} \int_{0}^{T}\left|\lambda\left(\boldsymbol{\varphi}_{s}\right) \dot{\boldsymbol{\varphi}}_{s}-\boldsymbol{b}\left(\boldsymbol{\varphi}_{s}\right)\right|^{2} d s: \boldsymbol{\varphi}_{0}=\boldsymbol{x}, \boldsymbol{\varphi}_{T}=\boldsymbol{y}, T \geq 0\right\}, \boldsymbol{x}, \boldsymbol{y} \in \mathbb{R}^{n}
$$

and by the extremals of this variational problem.

Let now the dynamical system $\dot{\boldsymbol{q}}_{t}=\boldsymbol{b}\left(\boldsymbol{q}_{t}\right)$ has a first integral. Let, say, $n=2$ and $\boldsymbol{b}(\boldsymbol{q})=\bar{\nabla} H(\boldsymbol{q})$ for some smooth generic function $H(\boldsymbol{q}), \boldsymbol{q} \in \mathbb{R}^{2}$, such that $\lim _{|\boldsymbol{q}| \rightarrow \infty} H(\boldsymbol{q})=$ $\infty$; in this case $H\left(\boldsymbol{q}_{t}\right) \equiv H\left(\boldsymbol{q}_{0}\right)$.

Assume again that the friction is strong: $\lambda^{\varepsilon}(\boldsymbol{q})=\varepsilon^{-1} \lambda(\boldsymbol{q})$. Make a time change $\widehat{\boldsymbol{q}}_{t}^{\varepsilon}=\boldsymbol{q}_{t / \varepsilon^{2}}$. Then

$$
\dot{\widehat{\boldsymbol{q}}}_{t}^{\varepsilon}=\frac{1}{\varepsilon^{2}} \nabla H\left(\widehat{\boldsymbol{q}}_{t}^{\varepsilon}\right)-\frac{\nabla \lambda\left(\widehat{\boldsymbol{q}}_{t}^{\varepsilon}\right)}{2 \lambda^{3}\left(\widehat{\boldsymbol{q}}_{t}^{\varepsilon}\right)}+\frac{1}{\lambda\left(\widehat{\boldsymbol{q}}_{t}^{\varepsilon}\right)} \dot{\boldsymbol{W}}_{t}, \widehat{\boldsymbol{q}}_{0}^{\varepsilon}=\boldsymbol{q} \in \mathbb{R}^{2} .
$$

Identify points of each connected component of every level set of $H(\boldsymbol{q})$. The set obtained after such an identification if homeomorphic in the natural topology to a graph $\Gamma$. Let $Y: \mathbb{R}^{2} \rightarrow \Gamma$ be the identification mapping. Then the long-time evolution of the system can be characterized by the stochastic process $\mathcal{Y}_{t}^{\varepsilon}=Y\left(\widehat{\boldsymbol{q}}_{t}^{\varepsilon}\right)$ on $\Gamma$. The process $\mathcal{Y}_{t}^{\varepsilon}$, in general, is not Markovian. But $\mathcal{Y}_{t}^{\varepsilon}$ converges weakly in the space of continuous functions $\varphi:[0, T] \rightarrow \Gamma$ as $\varepsilon \downarrow 0$ to a diffusion process on the graph $\Gamma$ ([9], Ch.8). This limiting process is defined by a family of second-order differential operators, one on each edge of $\Gamma$, and by the gluing conditions at the vertices. Following [9], one can evaluate these operators and the gluing conditions.

\subsection{Fast oscillating random friction in multidimensional case.}

Let us consider the case of fast oscillating in the space variable, random friction, in dimension $d \geq 2$. Let $(\Omega, \mathcal{F}, \mathbf{P})$ be a probability space. Let $\lambda(\boldsymbol{x}, \omega), \omega \in \Omega$ be a random field in $\mathbb{R}^{d}$ with the following properties:

(i) For any fixed $\omega \in \Omega$ and $\boldsymbol{x} \in \mathbb{R}^{d}$, the function $\infty>\Lambda \geq \lambda(\boldsymbol{x}, \omega) \geq \lambda_{0}>0$.

(ii) For every $\boldsymbol{x} \in \mathbb{R}^{d}$ the random variable $\lambda(\boldsymbol{x}, \omega)$ is independent of the Wiener process $\boldsymbol{W}_{t}$. 
(iii) The random field $\lambda(\boldsymbol{x}, \omega)$ has the form $\lambda(\boldsymbol{x}, \omega)=\lambda(T(\boldsymbol{x}) \omega)$ where $T(\boldsymbol{x}): \Omega \rightarrow \Omega$ is a $d$-dimensional dynamical system which preserves the measure $\mathbf{P}$ and is ergodic with respect to $\mathbf{P}$.

Let us now consider an analogue of (5.1):

$$
\mu \ddot{\boldsymbol{q}}_{t}^{\mu, \delta, \varepsilon}=-\lambda\left(\frac{\boldsymbol{q}_{t}^{\mu, \delta, \varepsilon}}{\varepsilon}, \omega\right) \dot{\boldsymbol{q}}_{t}^{\mu, \delta, \varepsilon}+\dot{\boldsymbol{W}}_{t}^{\delta}, \boldsymbol{q}_{0}^{\mu, \delta, \varepsilon}=\boldsymbol{q} \in \mathbb{R}^{d}, \dot{\boldsymbol{q}}_{0}^{\mu, \varepsilon, \delta}=\boldsymbol{p} \in \mathbb{R}^{d}
$$

For each fixed $\omega \in \Omega$, as we proved in Section 3, we have that $\boldsymbol{q}_{t}^{\mu, \delta, \varepsilon}(\omega)$ converges weakly to a process $\boldsymbol{q}_{t}^{\varepsilon}(\omega)$ as first $\mu \downarrow 0$ and then $\delta \downarrow 0$. The process $\boldsymbol{q}_{t}^{\varepsilon}$ is subject to

$$
\dot{\boldsymbol{q}}_{t}^{\varepsilon}=-\frac{1}{2 \varepsilon} \frac{\nabla \lambda\left(\frac{\boldsymbol{q}_{t}^{\varepsilon}}{\varepsilon}, \omega\right)}{\lambda^{3}\left(\frac{\boldsymbol{q}_{t}^{\varepsilon}}{\varepsilon}, \omega\right)}+\frac{1}{\lambda\left(\frac{\boldsymbol{q}_{t}^{\varepsilon}}{\varepsilon}, \omega\right)} \dot{\boldsymbol{W}}_{t}, \boldsymbol{q}_{0}^{\varepsilon}=\boldsymbol{q} \in \mathbb{R}^{d} .
$$

We conjecture that as $\varepsilon \downarrow 0, \boldsymbol{q}_{t}^{\varepsilon}$ converges weakly to a process $\boldsymbol{q}_{t}, \boldsymbol{q}_{0}=\boldsymbol{q} \in \mathbb{R}^{d}$ subject to the operator $\overline{\bar{L}}=\frac{1}{2} \sum_{i, j=1}^{d} \overline{\bar{a}}_{i j} \frac{\partial^{2}}{\partial x_{i} \partial x_{j}}$ with effective diffusivity

$$
\overline{\bar{a}}_{i j}=\mathbf{E}\left[\frac{\int_{\mathbb{T}^{d}} \frac{\partial N_{j}}{\partial y_{i}}(\boldsymbol{x}, \omega) \frac{1}{\lambda(\boldsymbol{x}, \omega)} d \boldsymbol{x}}{\int_{\mathbb{T}^{d}} \lambda(\boldsymbol{x}, \omega) d \boldsymbol{x}}+\delta_{i j} \frac{\int_{\mathbb{T}^{d}} \frac{1}{\lambda(\boldsymbol{x}, \omega)} d \boldsymbol{x}}{\int_{\mathbb{T}^{d}} \lambda(\boldsymbol{x}, \omega) d \boldsymbol{x}}\right] .
$$

Here the functions $N_{k}(\boldsymbol{x}, \omega)(1 \leq k \leq d)$ shall satisfy certain auxiliary problem. (We actually have a formulation of this problem but we are not sure about its validity: we let $N_{k}(\boldsymbol{x}, \omega)$ be the solution of the equation

$$
\mathbf{E}\left[\left(\nabla_{\boldsymbol{x}} N_{k}(\boldsymbol{x}, \omega)-\boldsymbol{e}_{k}\right) \cdot \nabla_{\boldsymbol{x}} \varphi(\boldsymbol{x}, \omega)\right]=0,
$$

for all $\varphi(\boldsymbol{x}, \omega)$ smooth and compactly supported in $\boldsymbol{x} \in \mathbb{R}^{d}$ and measurable with respect to $\omega \in \Omega$. The existence of solutions to this problem is guaranteed by the Lax-Milgram lemma.)

However, we are not aware of the validity of this conjecture nor a proof of it. We are also not sure about the correct reference of such a problem. (We thank E.Kosygina for pointing out to us two relevant papers [1] and [11].)

\subsection{Motion of charged particles in a magnetic field}

One can expect that using the regularization by smoothed white noise one can get the Smoluchowski-Kramers approximation for the equation

$$
\mu \ddot{\boldsymbol{q}}_{t}^{\mu}=\boldsymbol{b}\left(\boldsymbol{q}_{t}^{\mu}\right)-A\left(\boldsymbol{q}_{t}^{\mu}\right) \dot{\boldsymbol{q}}_{t}^{\mu}+\sigma \dot{\boldsymbol{W}}_{t}, \boldsymbol{q}_{0}^{\mu}=\boldsymbol{q}, \dot{\boldsymbol{q}}_{0}^{\mu}=\boldsymbol{p} ; \boldsymbol{q}, \boldsymbol{p} \in \mathbb{R}^{n}
$$


Here $\sigma>0$ and $A(\boldsymbol{q})$ are a matrix-valued functions having strictly positive eigenvalues for each $\boldsymbol{q} \in \mathbb{R}^{n}$. In particular, if $A(\boldsymbol{q})=\lambda(\boldsymbol{q}) A$, where $\lambda(\boldsymbol{q})>0$ and $A$ is a constant positive definite symmetric matrix, the problem can be reduced to the case considered in Section 3 by an appropriate linear change of variables.

If $A$ has a negative eigenvalue, the Smoluchowski-Kramers approximation is not applicable. The case of $A$ with pure imaginary eigenvalues is of interest since such equations describe the motion of charged particles in a magnetic field. If $\lambda=$ const, $n=2$ and $A=\left(\begin{array}{cc}0 & -1 \\ 1 & 0\end{array}\right)$, the problem was considered in [3]. In this case the SmoluchowskiKramers approximation holds after a regularization. If $\boldsymbol{b}(\boldsymbol{q})=-\nabla F(\boldsymbol{q}), \boldsymbol{q} \in \mathbb{R}^{2}$, and $A=\left(\begin{array}{cc}0 & -1 \\ 1 & 0\end{array}\right)$, one can show that the regularization by the smoothed white noise leads to the equation

$$
\boldsymbol{q}_{t}=\frac{1}{\lambda\left(\boldsymbol{q}_{t}\right)} \bar{\nabla} F\left(\boldsymbol{q}_{t}\right)-\sigma^{2} \frac{\bar{\nabla} \lambda(\boldsymbol{q})}{2 \lambda^{3}(\boldsymbol{q})}+\frac{\sigma}{\lambda(\boldsymbol{q})} \dot{\widetilde{\boldsymbol{W}}}_{t}, \boldsymbol{q}_{0}=\boldsymbol{q} \in \mathbb{R}^{2} .
$$

If the noise in $(6.5)$ is small $(0<\sigma<<1)$ the motion described by (6.6) has a fast and a slow components. Applying the results of [9], [2], one can describe the limiting (as $\sigma \downarrow 0$ ) slow component of $\widehat{\boldsymbol{q}}_{t}^{\sigma}=\boldsymbol{q}_{t / \sigma^{2}}$ as a diffusion process on the graph corresponding to the potential $F(\boldsymbol{q})$ (the graph is homeomorphic to the set of connected components of the level sets of $F(\boldsymbol{q})$ provided with the natural topology).

Acknowledgements: This work is supported in part by NSF Grants DMS-0803287 and DMS-0854982.

\section{References}

[1] G.Allaire, R.Orive, Homogenization of periodic non self-adjoint problems with large drift and potential, Esaim-control Optimisation and Calculus of Variations, vol. 13, no. 4, pp. 735-749, 2007.

[2] M.Brin, M.Freidlin, On stochastic behavior of perturbed Hamiltonian systems, Ergodic Theory and Dynamical Systems, 20 (2000), No.1. 55-76.

[3] S.Cerrai, M.Freidlin, Small mass asymptotics of a charged particle in a magnetic field and long-time influence of small perturbations, Journal of Statistical Physics, submitted.

[4] W.Feller, Generalized second order differential operators and their lateral conditions, Ill. J. Math., 1 (1957) pp.459-504.

[5] M.Freidlin, Some Remarks on the Smoluchowski-Kramers Approximation, Journal of Statistical Physics, 117, No.314, pp.617-634, 2004. 
[6] M.Freidlin, Functional Integration and Partial Differential Equations, Princeton University Press, 1985.

[7] M.Freidlin, Dirichlet's problem for an equation with periodic coefficients depending on a small parameter, Theory of Probability and Its Applications, 9, pp.133-139, 1964.

[8] M.Freidlin, A.Wentzell, Necessary and Sufficient Conditions for Weak Convergence of One-Dimensional Markov Processes. The Dynkin Festschrift: Markov Processes and their Applications, Birkhauser, (1994) pp.95-109.

[9] M.Freidlin, A.Wentzell, Random perturbations of dynamical systems, Springer, 1998.

[10] N.Ikeda, S.Watanabe, Stochastic Differential Equations and Diffusion Processes, second edition, North-Holland Publishing Company, 1989.

[11] M.Klepsyna, A.Piatnitski, Averaging of non-self adjoint parabolic equations with random evolution, Institut National de Recherche en Informatique et en Automatique, June 2000.

[12] N.Krylov, G-convergence of elliptic operators in non-divergence form, Math Zametki, 37, 4, pp. 522-527, April, 1985.

[13] G.Papanicolaou, S.R.S.Varadhan, Boundary value problems with rapidly oscillating random coefficients, Seria Coll. Math. Soc. János Bolyai, North Holland, Amsterdam, 1979.

[14] V.V.Zhikov, S.M.Kozlov, O.A.Oleinik, Homogenization of differential operators and integral functionals, Springer, 1994.

[15] V.V.Zhikov, S.M.Kozlov, O.A.Oleinik, and Kha T'en Ngoan, Averaging and G-convergence of differential operators, Russian Math Surveys, 34, 5, pp.65-133, 1979. 